\title{
Large gauge transformation, soft theorem, and Infrared divergence in inflationary spacetime
}

\author{
Takahiro Tanaka ${ }^{a}$ and Yuko Urakawa ${ }^{b}$ \\ ${ }^{a}$ Department of Physics, Kyoto University, \\ Sakyo, Kyoto 606-8502, Japan \\ ${ }^{b}$ Department of Physics and Astrophysics, Nagoya University, \\ Chikusa, Nagoya 464-8602, Japan \\ E-mail: tanaka@yukawa.kyoto-u.ac.jp, \\ urakawa.yuko@h.mbox.nagoya-u.ac.jp
}

ABSTRACT: It is widely known that the primordial curvature perturbation $\zeta$ has several universal properties in the infrared (IR) such as the soft theorem, which is also known as the consistency relation, and the conservation in time. They are valid in rather general single clock models of inflation. It has been argued that these universal properties are deeply related to the large gauge transformations in inflationary spacetime. However, the invariance under the large gauge transformations is not sufficient to show these IR properties. In this paper, we show that the locality condition is crucial to show the consistency relation and the conservation of $\zeta$. This argument also can apply to an interacting system with the inflaton and heavy fields which have arbitrary integer spins, including higher spin fields, which may be motivated from string theory. We will also show that the locality condition guarantees the cancellation of the IR divergences in a certain class of variables whose correlation functions resemble cosmologically observable quantities.

KeYwords: Effective Field Theories, Cosmology of Theories beyond the SM, Gauge Symmetry

ARXIV EPRINT: 1707.05485 


\section{Contents}

1 Introduction 1

2 Asymptotic symmetry and soft theorem 3

2.1 Large gauge transformations 4

2.1.1 Dilatation as a large gauge transformation 4

2.1.2 Two different prescriptions of dilatation 5

2.2 Dilatation invariance and Noether charge 6

2.3 Revisiting consistency relation $\quad 7$

$\begin{array}{lll}\text { 2.3.1 Condition for consistency relation } & 7\end{array}$

2.3.2 Conditions on the homogeneous mode and hard modes 9

3 Massive particles with arbitrary spins $\quad 16$

$\begin{array}{ll}3.1 & \text { Setup of the problem } \\ 3.2 & \text { Soft the }\end{array}$

$\begin{array}{lll}3.2 & \text { Soft theorem for heavy fields with non-zero spins } & 16\end{array}$

$\begin{array}{lll}3.3 & \text { Effective action } & 18\end{array}$

$\begin{array}{ll}3.3 .1 & \text { Influence functional } \\ 3.3 .2\end{array}$

$\begin{array}{ll}\text { 3.3.2 Soft theorem and effective action } & 19\end{array}$

$\begin{array}{ll}3.4 \text { Conservation of } \zeta & 21\end{array}$

$4 \quad$ IR divergences of inflationary correlators $\quad 23$

4.1 Overview of IR divergence problem 24

4.2 Cancellation of IR divergence and the locality condition 24

5 Relevance and irrelevance of soft graviton insertion $\quad 26$

6 Concluding remarks $\quad 28$

A Perturbative and non-perturbative definition of Noether charge $\quad 29$

$\begin{array}{ll}\text { B Computing the effective action } & 30\end{array}$

\section{Introduction}

The presence of a symmetry helps us to systematically understand various properties of a physical system. Understanding the physics based on the symmetry may open up the possibility of understanding a specific example based on a more universal property of physical systems which share similar symmetry groups. It has been suggested that the infrared (IR) properties of the primordial perturbations generated during inflation are deeply related to the large gauge transformations. The large gauge transformation is 
a local symmetry transformation which does not approach the unity at the infinity of spacetime $[1,2]$.

The large gauge transformations which play an important role in cosmology are those defined at a time constant slicing and diverge at the spatial infinity. In single field models of inflation, the invariance under the dilatation and shear transformations, which are both large gauge transformations, directly ensures the massless property of the curvature perturbation $\zeta$ and the gravitational waves $\gamma_{i j}$. It is widely known that these massless fields $\zeta$ and $\gamma_{i j}$ have several universal properties in the IR, which are valid in rather general single field models of inflation. First, at the tree-level computation, $\zeta$ and $\gamma_{i j}$ are non-linearly conserved in time in the IR limit [3-11]. (The conservation of $\zeta$ was discussed by including the radiative corrections of $\zeta$ in refs. $[12,13]$.) Second, the influence from the soft modes of $\zeta$ and $\gamma_{i j}$ on the hard modes are, rather generically, characterized by the so-called consistency relation $[14,15]$, which is an example of the soft theorem in cosmology. It has been suggested, e.g., in refs. [3, 16-20], that these universal properties are both consequences of the invariance under the large gauge transformations. However, it was also revealed that this invariance is not sufficient to derive the consistency relation. In refs. [18-20], the analyticity in the soft limit was additionally imposed to derive the consistency relation.

The consistency relation has been understood as a relation between the $n$-point correlation function with $n$ hard modes and the $(n+1)$-point correlation function with $n$ hard modes and one soft mode. In deriving the consistency relation, usually, the validity of the perturbation theory is presumed. However, this is not trivially guaranteed, because the massless fields $\zeta$ and $\gamma_{i j}$ are not screened in the large scale limit and their radiative corrections can diverge due to their unsuppressed IR contributions. Therefore, unless the IR divergence is regularized without violating the dilatation invariance, the consistency relation cannot be well-defined as a relation between the correlation functions. Notice that introducing a naive IR cutoff which regularizes the IR radiative corrections can violate the dilatation invariance, which is a crucial property for the consistency relation.

The goal of this paper is to clarify the relation between the large gauge transformations and the above-mentioned universal properties of $\zeta$ and $\gamma_{i j}$ in the IR, i.e., the soft theorem (also known as the consistency relation), the conservation in time, and the possible appearance of the IR divergence. In particular, along the line of the argument in refs. [18-20], we will clarify the physical meaning of the analyticity in the soft limit, which is needed to derive the consistency relation. In ref. [18], it was argued that this condition is related to the locality of theory. In this paper, we scrutinize this argument, clarifying what we need to require as the locality condition, because the locality has a broad meaning. We also discuss what the consistency relation actually describes, taking into account the possibility that the perturbative prediction can be spoiled by the IR divergence.

We will also show that the large gauge transformations play a crucial role also in discussing the radiative corrections from massive fields. Being motivated by string theory, which may predict the presence of higher spin fields in the four dimensional theory obtained after compactification, we consider massive fields with arbitrary integer spins. After deriving the consistency relation for the hard modes of the massive fields with non-zero spins, we discuss the condition that the curvature perturbation $\zeta$ stops evolving in time in 
the soft limit under the influence of the radiative corrections from the massive fields. This is a generalization of our previous study [19], where we considered the radiative corrections from a massive scalar field, to a massive field with a general integer spin. As was argued in ref. [21], exploring an imprint of the higher spin fields in the primordial non-Gaussianity of $\zeta$ may provide a unique probe of string theory. (See also refs. [22-25].) Our study provides the conditions for the absence of such an imprint made after the Hubble crossing time of the comoving scale of our interest during inflation.

Recently, the relation among the large gauge transformations, the soft theorem, and the IR divergence has been studied intensively for gauge theories in an asymptotically flat spacetime [26-28]. (For a review, see ref. [29].) In ref. [26], it was shown that Weinberg's soft theorem [30], which describes the influence of the soft photon and graviton, can be obtained as a Ward-Takahashi identity of the asymptotic symmetries at the null infinity. (The soft theorem for massless higher spin fields in an asymptotically flat spacetime was discussed in ref. [31].) More recently, (the cancellation of) the IR divergence was discussed, using the Noether charge of the asymptotic symmetries [32]. At first sight, the IR structures for the gauge fields in the asymptotically flat spacetime have similar properties to those for the primordial perturbations $\zeta$ and $\gamma_{i j}$ in cosmology. We give a closer look at this apparent similarity.

This paper is organized as follows. In section 2, considering single field models of inflation, we discuss the relation between the large gauge transformation and the consistency relation for $\zeta$. Here, we seek for a deeper understanding about the locality condition as the necessary condition to derive the consistency relation for $\zeta$. In section 3 , we will show that the discussion in section 2 can be straightforwardly extended to an interacting system composed of the inflaton and the massive fields with non-zero spins. We also show that the locality condition implies both the consistency relation for the hard modes of the massive fields and the conservation of $\zeta$. In section 4 , we show that the locality condition also ensures the cancellation of the IR divergence for a certain class of variables. In section 5, we briefly show that the discussion for the soft graviton proceeds almost in parallel to the one for the soft modes of $\zeta$, discussed in section 2-4. In section 6 , after summarizing our results, we discuss a similarity and an apparent difference between the IR properties for the gauge fields in the asymptotically flat spacetime and those for the primordial perturbations.

\section{Asymptotic symmetry and soft theorem}

In this section, we discuss the relation between the large gauge transformations and the soft theorem in cosmology, also known as the consistency relation. We clarify the condition that derives the consistency relation and discuss its physical meaning. Before we start our discussion, we clarify what we mean by "soft" and "hard." The soft modes mean the modes with $k / a H \rightarrow 0$ but $\boldsymbol{k} \neq 0$, and the hard modes mean the remaining inhomogeneous modes, including the super Hubble modes with $k / a H \lesssim 1$ but excluding the limit $k / a H \rightarrow 0$. By contrast, we describe the wave vector of a longer mode and that of a shorter mode as $\boldsymbol{k}_{L}$ and $\boldsymbol{k}_{S}$, respectively, simply based on the ratio between these wavenumbers, i.e., $k_{L} / k_{S} \ll 1$. 
As we will discuss in section 4 , in the limit $k / a H \ll 1$, a perturbative expansion can break down in computing some quantity and taking this limit requires a careful treatment.

\subsection{Large gauge transformations}

Likewise in the discussion about the soft photons and gravitons in the asymptotically flat spacetime, a large gauge transformation plays a crucial role for a clear understanding about the soft modes of $\zeta$ and $\gamma_{i j}$ in an inflationary spacetime. In line with refs. [1,2], we define the large gauge transformation as follows. A local symmetry denotes a symmetry under a transformation which is parametrized by a spacetime dependent function, while a global symmetry denotes a symmetry under a transformation by a spacetime independent function.

Among local symmetry transformations, it is important to make a distinction between small gauge transformation and large gauge transformations. The former becomes the identity at the infinity and the latter does not. In refs. [26, 29], it was shown that the soft theorem for the photons and the gravitons in the asymptotically flat spacetime can be derived from the Ward-Takahashi identities for large gauge transformations which do not vanish on $\mathcal{J}^{ \pm}$.

\subsubsection{Dilatation as a large gauge transformation}

First, let us clarify the prescription we adopt. In this paper, we use the ADM form of the line element:

$$
d s^{2}=-N^{2} d t^{2}+h_{i j}\left(d x^{i}+N^{i} d t\right)\left(d x^{j}+N^{j} d t\right),
$$

where we introduced the lapse function $N$, the shift vector $N^{i}$, and the spatial metric $h_{i j}$. We determine the time slicing, employing the uniform field gauge:

$$
\delta \phi=0 .
$$

We express the spatial metric $h_{i j}$ as

$$
h_{i j}=a^{2} e^{2 \zeta}\left[e^{\gamma}\right]_{i j},
$$

where $\gamma_{i j}$ is set to traceless. As spatial gauge conditions, we impose

$$
\partial^{i} \gamma_{i j}=0 .
$$

To discuss the soft modes of the primordial perturbations in the spatially flat FRW background, we consider the large gauge transformations, which do not vanish at the spatial infinity on a time constant surface. This large gauge transformation was first discussed in the context of cosmology by Weinberg in ref. [3]. In the unitary gauge, where the fluctuation of the inflaton vanishes, we consider, in particular, the dilatation:

$$
x^{i} \rightarrow e^{s} x^{i},
$$

where $s$ is a constant parameter. Under the dilatation, the curvature perturbation $\zeta$ transforms as

$$
\zeta(t, \boldsymbol{x}) \rightarrow \zeta_{s}(t, \boldsymbol{x})=\zeta\left(t, e^{-s} \boldsymbol{x}\right)-s .
$$


The change of $\zeta$ is given by

$$
\Delta_{s} \zeta(t, \boldsymbol{x})=-s\left(1+\boldsymbol{x} \cdot \partial_{\boldsymbol{x}} \zeta(t, \boldsymbol{x})\right)+\mathcal{O}\left(s^{2}\right) .
$$

The classical action in a diffeomorphism (Diff) invariant theory remains invariant under the transformation of $\zeta$ given in eq. (2.6). As one may expect from the fact that the dilatation shifts $\zeta$ by $-s$, the dilatation invariance is related to the massless property of $\zeta$, which implies that $\zeta$ is conserved at large scales in single clock inflation.

\subsubsection{Two different prescriptions of dilatation}

The dilatation invariance may be somehow confusing, because it also appears as a part of the de Sitter invariance by changing the time coordinate simultaneously. The Killing vector which corresponds to this transformation is given by

$$
-\eta \partial_{\eta}-x^{i} \partial_{i}
$$

where $\eta$ denotes the conformal time. The dilatation symmetry in de Sitter group states that the time shift can be compensated by the scale transformation. Since inflation has to end at some point, the time translation symmetry needs to be broken in the context of inflationary scenario.

The time translation symmetry is broken, when the physical frequency $\omega_{p h}$ becomes well below $\Lambda_{b}$ with $\Lambda_{b}^{4} \equiv \dot{\phi}^{2}$. In the effective field theory of inflation [33], the Goldstone mode, the pion $\pi$, is introduced to restore the invariance under the time reparametrization

$$
t \rightarrow t+\xi, \quad \pi \rightarrow \pi-\xi
$$

in the symmetry breaking phase. With this construction, the pion Lagrangian non-linearly preserves the invariance since $\pi$ appears only in the combination $t+\pi$. Through the coupling with the metric perturbations, the pion acquires the mass of $m_{\pi}=\mathcal{O}\left(\sqrt{\varepsilon_{1}} H\right)$.

The relation between the dilatation discussed in section 2.1.1 and the one discussed here is somewhat puzzling. As we will discuss in the following section, the former is preserved in an arbitrary quasi FRW spacetime, while the latter is a part of the de Sitter symmetry and is broken below the symmetry breaking scale $\Lambda_{b}$. Related to this point, preserving the former dilatation invariance directly ensures that $\zeta$ should be massless. On the other hand, there is no simple argument which shows the massless property of $\zeta$ in the latter prescription, where $\zeta$ is related to the Goldstone mode $\pi$ as $\zeta=-H \pi$. Related to this point, recall that the pion acquires the mass $m_{\pi}$ through the coupling with the metric perturbation for $\omega_{p h} \lesssim m_{\pi} \cdot{ }^{1}$ Therefore, in the regime where $\zeta$ approaches a constant value, the pion is no longer massless. The curvature perturbation is sometimes said to be a Goldstone mode, since $\pi=-\zeta / H$ is the Goldstone mode. This statement can cause a confusion, because it may sound as if $\zeta$ preserves the shift symmetry, being massless, because it is a Goldstone boson associated with the breaking of the de Sitter symmetry.

\footnotetext{
${ }^{1}$ Recall that the Goldstone mode for a global symmetry is not necessarily massless in a Lorentz violating background.
} 
In the following, by the dilatation, we mean the former one, which is a spatial coordinate transformation without the time coordinate change. Considering this dilatation, we discuss the IR behaviour of $\zeta$ such as the consistency relation and the conservation in time. We will emphasize that the invariance under this dilatation, which is a part of the large gauge transformations, is preserved as well in the quantized system and therefore there is no spontaneous symmetry breaking in this prescription.

\subsection{Dilatation invariance and Noether charge}

In this subsection, we discuss several implications of the dilatation invariance in single clock inflation. Following ref. [16], we define the Noether charge for the dilatation as

$$
Q_{\zeta} \equiv \frac{1}{2} \int d^{3} \boldsymbol{x}\left[\Delta_{s} \zeta(t, \boldsymbol{x}) \pi_{\zeta}(t, \boldsymbol{x})+\pi_{\zeta}(t, \boldsymbol{x}) \Delta_{s} \zeta(t, \boldsymbol{x})\right],
$$

where $\pi_{\zeta}$ denotes the conjugate momentum of $\zeta$, which satisfies

$$
\left[\zeta(t, \boldsymbol{x}), \pi_{\zeta}(t, \boldsymbol{y})\right]=i \delta(\boldsymbol{x}-\boldsymbol{y})
$$

Since the Hamiltonian for $\zeta$ is invariant under the dilatation, we obtain

$$
\left[Q_{\zeta}, H\right]=0
$$

which implies that $Q_{\zeta}$ is independent of time. The Noether charge is a generator of the dilatation transformation and satisfies

$$
\left[Q_{\zeta}, \zeta(x)\right]=-i \Delta_{s} \zeta(x)
$$

Using the Fourier components of the fields ${ }^{2}$ we can rewrite the Noether charge $Q_{\zeta}$ as

$$
Q_{\zeta}=-s \pi_{\zeta, \boldsymbol{k}=0}-\frac{s}{2} \int \frac{d^{3} \boldsymbol{k}}{(2 \pi)^{3}}\left\{\zeta_{\boldsymbol{k}}, \boldsymbol{k} \cdot \partial_{\boldsymbol{k}} \pi_{\zeta,-\boldsymbol{k}}\right\}+\mathcal{O}\left(s^{2}\right)
$$

In performing the Fourier transformation, we did not drop the surface term. Therefore, the charge $Q_{\zeta}$ given in eq. (2.9) is identical to the one given in eq. (2.13). The first term of $Q_{\zeta}$ only operates on the $\boldsymbol{k}=0$ mode. The Noether charge $Q_{\zeta}$ can diverge due to the IR modes, because it is an integral over the infinite spatial volume. In the following, we neglect higher order terms of $\mathcal{O}\left(s^{2}\right)$.

Equation (2.13) gives the non-perturbative definition of the Noether charge for the dilatation. Since an explicit computation usually relies on perturbative expansion in the interaction picture, one may want to introduce the Noether charge by using the fields in

\footnotetext{
${ }^{2}$ We use the convention of the Fourier transformation:

$$
f(\boldsymbol{x})=\int \frac{d^{3} \boldsymbol{k}}{(2 \pi)^{3}} e^{i \boldsymbol{k} \cdot \boldsymbol{x}} \hat{f}(\boldsymbol{k}), \quad \hat{f}(\boldsymbol{k})=\int d^{3} \boldsymbol{x} e^{-i \boldsymbol{k} \cdot \boldsymbol{x}} f(\boldsymbol{x}) .
$$

Here, the commutation relation for the Fourier modes of $\zeta$ and $\pi_{\zeta}$ is given by $\left[\zeta_{\boldsymbol{k}}, \pi_{\zeta \boldsymbol{k}^{\prime}}\right]=i(2 \pi)^{3} \delta\left(\boldsymbol{k}+\boldsymbol{k}^{\prime}\right)$.
} 
the interaction picture $a^{3}$

$$
Q_{\zeta}^{I} \equiv \frac{1}{2} \int d^{3} \boldsymbol{x}\left[\Delta_{s} \zeta^{I}(t, \boldsymbol{x}) \pi_{\zeta}^{I}(t, \boldsymbol{x})+\pi_{\zeta}^{I}(t, \boldsymbol{x}) \Delta_{s} \zeta^{I}(t, \boldsymbol{x})\right]
$$

where $\Delta_{s} \zeta^{I}(t, \boldsymbol{x})$ is given by

$$
\Delta_{s} \zeta^{I}(t, \boldsymbol{x})=-s\left(1+\boldsymbol{x} \cdot \partial_{\boldsymbol{x}} \zeta^{I}(t, \boldsymbol{x})\right)
$$

When we perform the dilatation transformation in the interaction picture or after the perturbative transformation, there is one caveat which should be kept in mind. Changing order of performing the finite dilatation transformation and performing the perturbative expansion leads us to a different answer. In order words, performing the dilatation in the interaction picture (with a use of $Q_{\zeta}^{I}$ ) and performing the dilatation in the Heisenberg picture (with $Q_{\zeta}$ ) are different, while this discrepancy disappears, when we consider the infinitesimal transformation generated by $d Q_{\zeta} /\left.d s\right|_{s \rightarrow 0}$. A lesson from here is that for a legitimate prescription, the dilatation transformation should be performed in the nonperturbative Heisenberg picture. A more detailed discussion can be found in appendix A.

\subsection{Revisiting consistency relation}

In this subsection, we discuss the condition(s) to derive the consistency relation of $\zeta$. We will see that while the dilatation invariance plays a crucial role, it is neither sufficient nor even necessary to derive the consistency relation.

\subsubsection{Condition for consistency relation}

The consistency relation for $\zeta$ was first derived by Maldacena from an explicit computation of the bi-spectrum of $\zeta$ [14]. Afterwards, the consistency relation was derived in a rather general setup of single field models of inflation [15], while several examples that do not satisfy the consistency relation were also reported [34-36] (see also refs. [37, 38]).

In ref. [14], it was argued that the bi-spectrum in the squeezed limit, which describes the correlation between the long mode $\boldsymbol{k}_{L}$ and short mode $\boldsymbol{k}_{S}$, can be computed by considering the influence of the long mode on the short mode. This influence can be described as the dilatation $\boldsymbol{k}_{S} \rightarrow e^{-\zeta_{L}} \boldsymbol{k}_{S}$. While $\zeta$ is not invariant under the dilatation transformation, ${ }^{4}$ we can construct another variable which is invariant under the dilatation transformation [39, 40]. In ref. [41], we showed that the leading contributions to the bi-spectrum of such an invariant variable in the squeezed limit are canceled, choosing the adiabatic vacuum. In this computation, the squeezed bi-spectrum of $\zeta$ satisfies Maldacena's consistency relation, which is the key to ensure the cancellation.

\footnotetext{
${ }^{3}$ Notice that since the free Hamiltonian $H_{0}$ is not invariant under the dilatation, i.e.,

$$
\left[H_{0}, Q_{\zeta}^{I}\right] \neq 0
$$

in contrast to $Q_{\zeta}$, which is time independent, the "charge" defined in the interaction picture $Q_{\zeta}^{I}$ varies

${ }^{4}$ The curvature perturbation $\zeta$ is invariant under the small gauge transformations but not under the large gauge transformations.
} in time. 
The consistency relation does not hold for an arbitrary quantum state even in single field models. In refs. [17, 18, 20], it has been directly and indirectly suggested that the dilatation invariance of the quantum state:

$$
Q_{\zeta}|\Psi\rangle=0
$$

is crucial to derive the consistency relation. In ref. [17], it was shown that the invariance of the 1PI effective action for $\zeta$ under the dilatation leads to a set of identities which relate the $n$-point function and the $(n+1)$-point function for $\zeta$. What we directly obtain from these identities, which are the so called Ward-Takahashi (WT) identity, is "the consistency relation," which relates the $n$-point function with $n$ hard modes to the $(n+1)$-point function with the homogeneous mode $\zeta_{\boldsymbol{k}=0}$ in addition to the $n$ hard modes. The only difference from the consistency relation is in that the inserted mode is not the soft mode $\zeta_{\boldsymbol{k}}$ with $\boldsymbol{k} \neq 0$.

Along this line, in refs. [20, 42, 43], "the consistency relation" was derived starting with the dilatation invariance of the wave function in the $\zeta$ representation, $\Psi[\zeta] \equiv\langle\zeta \mid \Psi\rangle$, where $|\zeta\rangle$ is the normalized eigenstate of $\zeta$, i.e.,

$$
\Psi\left[\zeta_{s}(t, \boldsymbol{x})\right]=\Psi[\zeta(t, \boldsymbol{x})],
$$

which directly follows from the invariance of the quantum state under the dilatation $(2.17) .^{5}$ If and only if the WT identity which describes the insertion of $\zeta_{\boldsymbol{k}=0}$ can be extended to the relation which describes the insertion of the soft mode $\zeta_{\boldsymbol{k}}$ with $\boldsymbol{k} \neq 0$, we obtain the consistency relation:

$$
\lim _{k_{n} \rightarrow 0} \frac{\mathcal{C}^{(n)}\left(\left\{\boldsymbol{k}_{i}\right\}_{n}\right)}{P\left(k_{n}\right)}=-\left(\sum_{i=2}^{n-1} \boldsymbol{k}_{i} \cdot \partial_{\boldsymbol{k}_{i}}+3(n-2)\right) \mathcal{C}^{(n-1)}\left(\left\{\boldsymbol{k}_{i}\right\}_{n-1}\right)
$$

where $\mathcal{C}^{(n)}$ denotes the $n$-point function of $\zeta$ with the momentum conservation factor

$$
(2 \pi)^{3} \delta\left(\sum_{i=1}^{n} \boldsymbol{k}_{i}\right)
$$

removed. Since we additionally need to impose that this extension is possible, requesting the dilatation invariance of the quantum state (2.17) is not enough to derive the consistency relation.

Now, the question is "What is the physical meaning of the additional condition that allows the WT identity to be smoothly extended to the consistency relation, which describes the insertion of the soft mode?" This issue was first addressed in ref. [18], where it was argued that this condition is related to the locality of the theory. Even if the original theory is local, the Lagrangian density for $\zeta$ in the unitary gauge becomes non-local due to

${ }^{5}$ For instance, at $\mathcal{O}(s)$, eq. (2.18) gives

$$
0=\int d^{3} \boldsymbol{x} \Delta_{s} \zeta(t, \boldsymbol{x}) \frac{\delta}{\delta \zeta(t, \boldsymbol{x})} \psi[\zeta]
$$

which can be obtained from $0=\left\langle\zeta(x)\left|Q_{\zeta}\right| \psi\right\rangle$ by inserting the expression of the Noether charge, given in eq. (2.9). Here, we used $\left\langle\zeta\left|\pi_{\zeta}\right| \psi\right\rangle \propto \delta \psi[\zeta] / \delta \zeta$. 
the presence of the Lagrange multipliers, the lapse function and the shift vector, which are given by solving the elliptic equations. For instance, at the linear order in perturbation, the shift vector $N_{i}$ includes a contribution given by

$$
\partial_{i} N_{i} \supset \varepsilon \dot{\zeta},
$$

which introduces non-local interaction vertices. In the standard slow-roll inflation, $\dot{\zeta}_{k}$ is suppressed in the limit $\boldsymbol{k} \rightarrow 0$ as $\dot{\zeta}_{\boldsymbol{k}}=\mathcal{O}\left(k^{p} \zeta_{\boldsymbol{k}}\right)$ with $p \geq 1$. However, in the absence of this suppression, the coefficients of the non-local interaction vertices in the Fourier space can be singular in the limit $\boldsymbol{k} \rightarrow 0$. Then, $N_{i}$ should be determined discontinuously at $\boldsymbol{k}=0$ in order to avoid the singular behaviour.

Along this line, our purpose of this section is to sharpen the relation between the condition for the locality and the condition of being able to extend the "consistency relation" with the insertion of the homogeneous mode to the consistency relation with the soft mode $\boldsymbol{k} \neq 0$, clarifying the physical meaning of the condition. As was mentioned above, the Lagrangian density for $\zeta$ (and also for the gravitational waves) is non-local in the sense that the Lagrangian density cannot be solely determined by the dynamical fields at each spacetime point. For a classical theory, the condition for the smooth extension of the homogeneous mode $\boldsymbol{k}=0$ to the soft modes $\boldsymbol{k} \neq 0$ with a suitable fall-off at the spatial infinity is nothing but the one to pick up the Weinberg's adiabatic mode [3]. We elaborate the physical meaning of this condition for a quantum theory by using the Noether charge $Q_{\zeta}$. As will be discussed in the next section, with the use of the Noether charge, a generalization to the case with higher spin fields proceeds straightforwardly.

\subsubsection{Conditions on the homogeneous mode and hard modes}

As was mentioned in section 2.3.1, the validity of the consistency relation is deeply related to the invariance of the wave function or the effective action under the dilatation, which is a large gauge transformation. Maldacena derived the consistency relation for the tree-level bi-spectrum in the squeezed limit, i.e., (2.19) with $n=3$, choosing the adiabatic vacuum (or the Euclidean vacuum) [14]. This vacuum also can be defined non-perturbatively by requesting the regularity of correlation functions in the limits $t \rightarrow-\infty(1 \pm i \epsilon){ }^{6}$ Here, the time path is rotated towards the imaginary axis in the distant past. This serves one of the examples of the quantum state $|\Psi\rangle$, which preserves the dilatation invariance, since this definition does not artificially introduce any specific scale. At perturbative level, a correlation function for the Euclidean vacuum can be calculated by adopting the $i \epsilon$ prescription. Here, the (free) mode function should be chosen to be the one for the adiabatic vacuum (a.k.a, the Bunch-Davies vacuum in de Sitter limit) [44].

In order to study the condition for the dilatation invariance of the quantum state (2.17), we decompose the wave function in terms of the eigenstates of the spatial average of $\zeta$ all over a time constant slicing, e.g., at the end of inflation,

$$
\bar{\zeta} \equiv \frac{\int d^{3} \boldsymbol{x} \zeta(\boldsymbol{x})}{\int d^{3} \boldsymbol{x}}
$$

\footnotetext{
${ }^{6}$ To be more precise, the regularity should be required in the limits $t \rightarrow-\infty(1 \pm i \epsilon)$ for a fixed set of momenta. Therefore, all the momenta go to the UV in these limits.
} 
as

$$
|\Psi\rangle=\int d \bar{\zeta}^{c}\left|\psi\left(\bar{\zeta}^{c}\right)\right|\left|\bar{\zeta}^{c}\right\rangle|\Psi\rangle_{\bar{\zeta}^{c}}
$$

The eigenstate $\left|\bar{\zeta}^{c}\right\rangle$ satisfies

$$
\bar{\zeta}\left|\bar{\zeta}^{c}\right\rangle=\bar{\zeta}^{c}\left|\bar{\zeta}^{c}\right\rangle
$$

where $\bar{\zeta}^{c}$ is a c-number eigenvalue. In order to distinguish the eigenvalues of $\bar{\zeta}$, which are $c$-numbers, from the operator $\bar{\zeta}$, we put the index $c$ on the eigenvalues. In eq. (2.21), we factorized the wave function of $\bar{\zeta}, \psi\left(\bar{\zeta}^{c}\right)$, from $\left\langle\bar{\zeta}^{c} \mid \Psi\right\rangle$, while absorbing the phase into $|\Psi\rangle_{\bar{\zeta}^{c}}$, as

$$
\left\langle\bar{\zeta}^{c} \mid \Psi\right\rangle=\left|\psi\left(\bar{\zeta}^{c}\right)\right||\Psi\rangle_{\bar{\zeta}^{c}}
$$

From the normalization condition for $|\Psi\rangle_{\bar{\zeta}^{c}}$, the amplitude of the wave function $\left|\psi\left(\bar{\zeta}^{c}\right)\right|$ is unambiguously defined. Since the quantum state $|\Psi\rangle$ also includes the inhomogeneous modes with $\boldsymbol{k} \neq 0,\left\langle\bar{\zeta}^{c} \mid \Psi\right\rangle$ should be understood as a vector in infinite dimensional Hilbert space. We express the normalized quantum state for all the modes with $\boldsymbol{k} \neq 0$ obtained by the projection of $|\Psi\rangle$ to the eigen state $\left|\bar{\zeta}^{c}\right\rangle$ as $|\Psi\rangle_{\bar{\zeta}^{c}}$. Using eq. (2.12), we obtain

$$
\left[i Q_{\zeta}, \bar{\zeta}\right]=-s \text {. }
$$

Since an operation of $e^{i Q_{\zeta}}$ shifts the eigenvalue of $\bar{\zeta}$ by $s$, i.e.,

$$
e^{i Q_{\zeta}}\left|\bar{\zeta}^{c}\right\rangle=\left|\bar{\zeta}^{c}+s\right\rangle
$$

we obtain

$$
i Q_{\zeta}\left|\bar{\zeta}^{c}\right\rangle=s \frac{\partial}{\partial \bar{\zeta}^{c}}\left|\bar{\zeta}^{c}\right\rangle
$$

Using this condition, we can express the dilatation invariance condition for the quantum state $|\Psi\rangle,(2.17)$, as

$$
0=\int d \bar{\zeta}^{c}\left[-s \frac{\partial\left|\psi\left(\bar{\zeta}^{c}\right)\right|}{\partial \bar{\zeta}^{c}}\left|\bar{\zeta}^{c}\right\rangle|\Psi\rangle_{\bar{\zeta}^{c}}+\left|\psi\left(\bar{\zeta}^{c}\right)\right|\left|\bar{\zeta}^{c}\right\rangle\left(i Q_{\zeta}-s \frac{\partial}{\partial \bar{\zeta}^{c}}\right)|\Psi\rangle_{\bar{\zeta}^{c}}\right]
$$

Operating $\left\langle\bar{\zeta}^{c^{\prime}}\right|$ on eq. (2.27), the real and imaginary parts, respectively, give

$$
\frac{\partial}{\partial \bar{\zeta}^{c}}\left|\psi\left(\bar{\zeta}^{c}\right)\right|=0
$$

and

$$
i Q_{\zeta}|\Psi\rangle_{\bar{\zeta}^{c}}=s \frac{\partial}{\partial \bar{\zeta}^{c}}|\Psi\rangle_{\bar{\zeta}^{c}}
$$

The first condition (2.28) requires that the amplitude of the wave function $\psi\left(\bar{\zeta}^{c}\right)$, which represents the probability distribution of $\bar{\zeta}$, should be flat in the direction of $\bar{\zeta}$ in the Hilbert space. The condition (2.28) is satisfied, e.g., for a Gaussian wave function whose variance 
blows up in the limit of the homogeneous mode, which is the case for a nearly scale invariant power spectrum. While the wave function for $\bar{\zeta}$ becomes non-normalizable, as will be discussed in section 4 , this does not create any problems as far as we compute a certain set of quantities which mimic to observable quantities. The condition (2.28) requires that the probability distribution should be non-perturbatively flat in the direction of the homogeneous mode $\bar{\zeta}$. The second condition may be a little more non-trivial. The quantum state of the inhomogeneous modes generically changes under a variation of the homogeneous mode $\bar{\zeta}$. The condition (2.29) restricts how the quantum state of the inhomogeneous modes should respond to the change of the homogeneous mode, which is expressed by the operation of $Q_{\zeta}$.

Since the consistency relation describes an insertion of an inhomogeneous mode with $\boldsymbol{k} \neq 0$ that suitably falls off at the spatial infinity, we need to extend the above argument to inhomogeneous modes. For this purpose, we introduce the generator of a spatial dependent dilatation given by

$$
Q_{\zeta}^{W}(\boldsymbol{x}) \equiv \frac{1}{2} \int d^{3} \boldsymbol{x}^{\prime} W\left(\boldsymbol{x}^{\prime}-\boldsymbol{x}\right)\left[\Delta_{s} \zeta\left(t, \boldsymbol{x}^{\prime}\right) \pi_{\zeta}\left(t, \boldsymbol{x}^{\prime}\right)+\pi_{\zeta}\left(t, \boldsymbol{x}^{\prime}\right) \Delta_{s} \zeta\left(t, \boldsymbol{x}^{\prime}\right)\right]
$$

where $W(\boldsymbol{x})$ denotes a smooth window function which is normalized as $\int d^{3} \boldsymbol{x} W(\boldsymbol{x})=1$ and which vanishes at $|\boldsymbol{x}| \gtrsim L$. Here, we set $L$ to be of order of $1 / k_{L}$. The generator $Q_{\zeta}^{W}(\boldsymbol{x})$ induces the dilatation only for the fields at $\boldsymbol{x}^{\prime}$ with $\left|\boldsymbol{x}-\boldsymbol{x}^{\prime}\right| \lesssim L$. The Noether charge (2.9) is defined by the integral all over the time constant slicing and hence the integral does not converge in general. The introduction of the window function can make the integral converge. Since the domain of integration is finite, the generator $Q_{\zeta}^{W}(\boldsymbol{x})$ depends on where we choose the center of the integral domain, $\boldsymbol{x}$. Performing the Fourier transformation, we obtain

$$
Q_{\zeta}^{W}\left(\boldsymbol{k}_{L}\right)=-s_{-\boldsymbol{k}_{L}}\left[\pi_{\boldsymbol{k}_{L}}+\frac{1}{2} \int \frac{d^{3} \boldsymbol{k}}{(2 \pi)^{3}}\left\{\zeta_{\boldsymbol{k}}, \boldsymbol{k} \cdot \partial_{\boldsymbol{k}} \pi_{\boldsymbol{k}_{L}-\boldsymbol{k}}\right\}\right]+\mathcal{O}\left(s^{2}\right)
$$

where $\hat{W}(\boldsymbol{k})$ denotes the Fourier mode of the window function, which is normalized as

$$
\lim _{\boldsymbol{k} \rightarrow 0} \hat{W}(\boldsymbol{k})=1
$$

and vanishes for $k \gg 1 / L$. Here, we introduced

$$
s_{\boldsymbol{k}} \equiv s \hat{W}(\boldsymbol{k}) .
$$

We focus on the field within a large volume of $O\left(L_{c}^{3}\right)$. We set $L_{c}$ to be much larger than all the wavelengths, i.e., $L_{c} \gg 1 / k_{L}, 1 / k_{S}$, and we will send $L_{c} \rightarrow \infty$ after our computation. Next, we introduce a smeared field in momentum space defined by

$$
\tilde{\zeta}_{\boldsymbol{k}_{L}} \equiv L_{c}^{3} \int d^{3} \boldsymbol{k}^{\prime} \hat{\mathcal{W}}\left(\boldsymbol{k}_{L}-\boldsymbol{k}^{\prime}\right) \zeta_{\boldsymbol{k}^{\prime}},
$$

with

$$
\hat{\mathcal{W}}(\boldsymbol{k})=\prod_{i=1}^{3} \theta\left[\left(2 L_{c}\right)^{-1}-\left|k_{i}\right|\right] .
$$


$\tilde{\zeta}_{\boldsymbol{k}_{L}}$ describes a collective mode with the representative wavenumber $\boldsymbol{k}_{L}$ and when we evaluate $\tilde{\zeta}$ in the position space by performing the inverse Fourier transformation, it decays outside the local volume of $O\left(L_{c}^{3}\right)$. The fluctuations outside the local volume can be described as the modes which are orthogonal to the collective mode $\tilde{\zeta}_{\boldsymbol{k}_{L}}$. Since the correlations between the fluctuations for $|\boldsymbol{x}| \ll L_{c}$ and those outside the local volume of $O\left(L_{c}^{3}\right)$ are negligibly small, in what follows, we neglect the fluctuations outside the local volume, which will disappear after taking the limit $L_{c} \rightarrow \infty$.

The commutation relation of $Q_{\zeta}^{W}$ with the long mode $\zeta_{\boldsymbol{k}_{L}}$ is given by

$$
\left[i Q_{\zeta}^{W}\left(\boldsymbol{k}_{L}\right), \tilde{\zeta}_{\boldsymbol{p}_{L}}\right]=-\left(2 \pi L_{c}\right)^{3} s_{-\boldsymbol{k}_{L}} \hat{\mathcal{W}}\left(\boldsymbol{k}_{L}+\boldsymbol{p}_{L}\right)
$$

Equation (2.35) states that the generator $Q_{\zeta}^{W}\left(\boldsymbol{k}_{L}\right)$ shifts the collective soft mode $\tilde{\zeta}_{\boldsymbol{k}_{L}}$ by $-s_{\boldsymbol{k}_{L}}\left(2 \pi L_{c}\right)^{3}$. We will find that the factor $\left(2 \pi L_{c}\right)^{3}$, which blows up in the limit $L_{c} \rightarrow \infty$, is cancelled out in the final expression of the consistency relation as it should be. Whilst, the commutation relation with the short mode $\zeta_{\boldsymbol{p}_{S}}$ is given by

$$
\left[i Q_{\zeta}^{W}\left(\boldsymbol{k}_{L}\right), \zeta_{\boldsymbol{p}_{S}}\right] \simeq s_{-\boldsymbol{k}_{L}} \partial_{\boldsymbol{p}_{S}}\left(\boldsymbol{p}_{S} \zeta_{\boldsymbol{p}_{S}+\boldsymbol{k}_{L}}\right),
$$

where we approximated $\boldsymbol{k}_{S}+\boldsymbol{k}_{L}$ as $\boldsymbol{k}_{S}$.

Repeating the argument around eqs. (2.21)-(2.23) except that the homogeneous mode $\bar{\zeta}$ is now replaced with a collective inhomogeneous soft modes $\tilde{\zeta}_{\boldsymbol{k}_{L}}$, we expand the quantum state $|\Psi\rangle$ in terms of the orthonormal basis $\left\{\left|\tilde{\zeta}_{\boldsymbol{p}_{L}}^{c}\right\rangle\right\}$, which are the eigenstates of $\tilde{\zeta}_{\boldsymbol{p}_{L}}$, as

$$
|\Psi\rangle=\int d \tilde{\zeta}_{\boldsymbol{p}_{L}}^{c}\left|\psi\left(\tilde{\zeta}_{\boldsymbol{p}_{L}}^{c}\right)\right|\left|\tilde{\zeta}_{\boldsymbol{p}_{L}}^{c}\right\rangle|\Psi\rangle_{\tilde{\zeta}_{\boldsymbol{p}_{L}}^{c}}
$$

where we factorized the amplitude of the wave function, $\left|\psi\left(\tilde{\zeta}_{\boldsymbol{k}_{L}}^{c}\right)\right|$, from $\left\langle\tilde{\zeta}_{\boldsymbol{k}_{L}}^{c} \mid \Psi\right\rangle$, as before. Using eq. (2.35), we obtain

$$
i Q_{\zeta}^{W}\left(\boldsymbol{k}_{L}\right)\left|\tilde{\zeta}_{-\boldsymbol{k}_{L}}^{c}\right\rangle=\left(2 \pi L_{c}\right)^{3} s_{-\boldsymbol{k}_{L}} \frac{\partial}{\partial \tilde{\zeta}_{-\boldsymbol{k}_{L}}^{c}}\left|\tilde{\zeta}_{-\boldsymbol{k}_{L}}^{c}\right\rangle .
$$

As we have already discussed, the dilatation invariance requires the conditions (2.28) and (2.29). In particular, the second condition (2.29) restricts how the inhomogeneous modes respond to the insertion of the homogeneous mode $\bar{\zeta}$. In the following, we will show that when this condition can be extended to the soft mode with $\boldsymbol{k}_{L} \neq 0$, i.e.,

$$
i Q_{\zeta}^{W}\left(\boldsymbol{k}_{L}\right)|\Psi\rangle_{\tilde{\zeta}_{-\boldsymbol{k}_{L}}^{c}}=\left(2 \pi L_{c}\right)^{3} s_{-\boldsymbol{k}_{L}} \frac{\partial}{\partial \tilde{\zeta}_{-\boldsymbol{k}_{L}}^{c}}|\Psi\rangle_{\tilde{\zeta}_{-\boldsymbol{k}_{L}}^{c}}
$$

is fulfilled, we can derive the consistency relation for $\zeta$ as shown below.

In order to show the consistency relation by using the condition (2.39), we evaluate

$$
\left\langle\Psi\left|\left[i Q_{\zeta}^{W}\left(\boldsymbol{k}_{L}\right), \zeta_{\boldsymbol{k}_{S 1}} \cdots \zeta_{\boldsymbol{k}_{S n}}\right]\right| \Psi\right\rangle
$$

in two ways: first by operating $i Q_{\zeta}^{W}\left(\boldsymbol{k}_{L}\right)$ on the quantum state $|\Psi\rangle$ and second by considering the change of the short wavelength modes $\zeta_{\boldsymbol{k}_{S}}$ under the inhomogeneous dilatation, 
expressed by the commutation relation with $i Q_{\zeta}^{W}\left(\boldsymbol{k}_{L}\right)$. When the condition (2.39) is satisfied, using eq. (2.38), we obtain

$$
\begin{aligned}
i Q_{\zeta}^{W}\left(\boldsymbol{k}_{L}\right)|\Psi\rangle & =\left(2 \pi L_{c}\right)^{3} s_{-\boldsymbol{k}_{L}} \int d \tilde{\zeta}_{-\boldsymbol{k}_{L}}^{c}\left|\psi\left(\tilde{\zeta}_{-\boldsymbol{k}_{L}}^{c}\right)\right| \frac{\partial}{\partial \tilde{\zeta}_{-\boldsymbol{k}_{L}}^{c}}\left(\left|\tilde{\zeta}_{-\boldsymbol{k}_{L}}^{c}\right\rangle|\Psi\rangle_{\tilde{\zeta}_{-\boldsymbol{k}_{L}}^{c}}\right) \\
& =-\left(2 \pi L_{c}\right)^{3} s_{-\boldsymbol{k}_{L}} \int d \tilde{\zeta}_{-\boldsymbol{k}_{L}}^{c} \frac{\partial\left|\psi\left(\tilde{\zeta}_{-\boldsymbol{k}_{L}}\right)\right|}{\partial \tilde{\zeta}_{-\boldsymbol{k}_{L}}^{c}}\left|\tilde{\boldsymbol{\zeta}}_{-\boldsymbol{k}_{L}}^{c}\right\rangle|\Psi\rangle_{\tilde{\zeta}_{-\boldsymbol{k}_{L}}^{c}}
\end{aligned}
$$

When we neglect the non-linear contributions of the soft modes, ${ }^{7}$ the wave function of $\tilde{\zeta}_{\boldsymbol{k}_{L}}^{c}$ is given by the Gaussian distribution function. Since the square of $\left|\psi\left(\tilde{\zeta}_{\boldsymbol{k}_{L}}^{c}\right)\right|$ gives the probability distribution, we can express the amplitude of the Gaussian wave function as

$$
\left|\psi\left(\tilde{\zeta}_{-\boldsymbol{k}_{L}}^{c}\right)\right| \propto \exp \left(-\frac{\tilde{\zeta}_{\boldsymbol{k}_{L}}^{c} \tilde{\zeta}_{-\boldsymbol{k}_{L}}^{c}}{4\left(2 \pi L_{c}\right)^{3} P_{\zeta}\left(k_{L}\right)}\right)
$$

where $P_{\zeta}\left(k_{L}\right)$ denotes the power spectrum of $\zeta$. This is because the variance of $\tilde{\zeta}_{\boldsymbol{k}_{L}}^{c}$ is given by

$$
\left\langle\left|\tilde{\zeta}_{\boldsymbol{k}_{L}}\right|^{2}\right\rangle=(2 \pi)^{3} L_{c}^{6} \int d^{3} \boldsymbol{k} \hat{\mathcal{W}}\left(\boldsymbol{k}_{L}-\boldsymbol{k}\right) \hat{\mathcal{W}}\left(-\boldsymbol{k}_{L}+\boldsymbol{k}\right) P_{\zeta}\left(k_{L}\right) \simeq\left(2 \pi L_{c}\right)^{3} P_{\zeta}\left(k_{L}\right),
$$

where the last equality is exact in the limit $L_{c} \rightarrow \infty$. Remember that $\tilde{\zeta}_{\boldsymbol{k}_{L}}^{c}$ is not an independent variable from $\tilde{\zeta}_{-\boldsymbol{k}_{L}}^{c}$, since $\tilde{\zeta}_{\boldsymbol{k}_{L}}^{c}=\tilde{\zeta}_{-\boldsymbol{k}_{L}}^{c *}$ holds from the reality of $\zeta$.

Using eq. (2.42), we obtain

$$
\frac{\partial}{\partial \tilde{\zeta}_{-\boldsymbol{k}_{L}}^{c}}\left|\psi\left(\tilde{\zeta}_{-\boldsymbol{k}_{L}}^{c}\right)\right| \stackrel{\text { s.l. }}{\approx}-\frac{\tilde{\zeta}_{\boldsymbol{k}_{L}}^{c}}{2\left(2 \pi L_{c}\right)^{3} P_{\zeta}\left(k_{L}\right)}\left|\psi\left(\tilde{\zeta}_{-\boldsymbol{k}_{L}}^{c}\right)\right| .
$$

Here and hereafter, we use $\stackrel{\text { s.l. }}{\approx}$ to express that we approximate the wave function of the soft mode by the above Gaussian distribution function. Replacing the eigenvalue $\tilde{\zeta}_{\boldsymbol{k}_{L}}^{c}$ with the operator $\tilde{\zeta}_{\boldsymbol{k}_{L}}$, which commutes with the integral over $\tilde{\zeta}_{\boldsymbol{k}_{L}}^{c}$, we obtain

$$
i Q_{\zeta}^{W}\left(\boldsymbol{k}_{L}\right)|\Psi\rangle \stackrel{\text { s.l. }}{\approx} \frac{s_{-\boldsymbol{k}_{L}}}{2 P_{\zeta}\left(k_{L}\right)} \tilde{\zeta}_{\boldsymbol{k}_{L}}|\Psi\rangle
$$

Using this expression, we arrive at

$$
\left\langle\Psi\left|\left[i Q_{\zeta}^{W}\left(\boldsymbol{k}_{L}\right), \zeta_{\boldsymbol{k}_{S 1}} \cdots \zeta_{\boldsymbol{k}_{S n}}\right]\right| \Psi\right\rangle \stackrel{\text { s.l. }}{\approx}-\frac{s_{-\boldsymbol{k}_{L}}}{P_{\zeta}\left(k_{L}\right)}\left\langle\Psi\left|\tilde{\zeta}_{\boldsymbol{k}_{L}} \zeta_{\boldsymbol{k}_{S 1}} \cdots \zeta_{\boldsymbol{k}_{S n}}\right| \Psi\right\rangle
$$

Meanwhile, using eq. (2.36), we obtain

$$
\left\langle\Psi\left|\left[i Q_{\zeta}^{W}\left(\boldsymbol{k}_{L}\right), \zeta_{\boldsymbol{k}_{S 1}} \cdots \zeta_{\boldsymbol{k}_{S n}}\right]\right| \Psi\right\rangle=s_{-\boldsymbol{k}_{L}} \sum_{i=1}^{n} \partial_{\boldsymbol{k}_{S i}} \boldsymbol{k}_{S i}\left\langle\Psi\left|\zeta_{\boldsymbol{k}_{S 1}} \cdots \zeta_{\boldsymbol{k}_{S n}}\right| \Psi\right\rangle .
$$

\footnotetext{
${ }^{7}$ Here, all the interaction vertexes connected to more than two soft modes are neglected.
} 
Equating these two expressions derived from the two different ways, we have

$$
-\left\langle\Psi\left|\tilde{\zeta}_{\boldsymbol{k}_{L}} \zeta_{\boldsymbol{k}_{S 1}} \cdots \zeta_{\boldsymbol{k}_{S n}}\right| \Psi\right\rangle \stackrel{\text { s.l. }}{\approx} P_{\zeta}\left(k_{L}\right) \sum_{i=1}^{n} \partial_{\boldsymbol{k}_{S i}} \boldsymbol{k}_{S i}\left\langle\Psi\left|\zeta_{\boldsymbol{k}_{S 1}} \cdots \zeta_{\boldsymbol{k}_{S n}}\right| \Psi\right\rangle .
$$

The ordinary form of the consistency relation (2.19) is the one obtained by removing the delta function, which describes the momentum conservation, from the expression

$$
-\left\langle\Psi\left|\zeta_{\boldsymbol{p}_{L}} \zeta_{\boldsymbol{k}_{S 1}} \cdots \zeta_{\boldsymbol{k}_{S n}}\right| \Psi\right\rangle \stackrel{\text { s.l. }}{\approx} P_{\zeta}\left(p_{L}\right) \sum_{i=1}^{n} \partial_{\boldsymbol{k}_{S i}} \boldsymbol{k}_{S i}\left\langle\Psi\left|\zeta_{\boldsymbol{k}_{S 1}} \cdots \zeta_{\boldsymbol{k}_{S n}}\right| \Psi\right\rangle .
$$

If we take the average of eq. (2.49) operating $L_{c}^{3} \int d^{3} \boldsymbol{p}_{L} \hat{\mathcal{W}}\left(\boldsymbol{k}_{L}-\boldsymbol{p}_{L}\right)$, we recover eq. (2.48). After we take the limit $L_{c} \rightarrow \infty$, which is automatically required when we take the soft limit $\boldsymbol{k}_{L} \rightarrow 0$, the averaging window becomes infinitesimally narrow. Therefore, we can conclude that eq. (2.48) is equivalent to the ordinary consistency relation.

Under the Gaussian approximation of $\left|\psi\left(\tilde{\zeta}_{\boldsymbol{k}_{L}}^{c}\right)\right|$, the only assumption imposed to derive the consistency relation (2.19) is eq. (2.39), which states that the influence of inhomogeneous dilatation is identical to shifting the collective soft mode $\tilde{\zeta}_{\boldsymbol{k}_{L}}$ which interacts with the hard modes by $+\left(2 \pi L_{c}\right)^{3} s_{\boldsymbol{k}_{L}}$. (The factor $\left(2 \pi L_{c}\right)^{3}$ here is an artifact caused by discussing the Fourier space collective mode.) The consistency relation can be obtained, when the condition (2.29), which was required to preserve the dilatation invariance of the quantum state, can be extended to the inhomogeneous soft mode with $\boldsymbol{k}_{L} \neq 0$. In this sense, the condition (2.39) can be understood as a quantum version of the condition for Weinberg's adiabatic mode.

The condition (2.39) cannot be satisfied, in case the (linear) soft mode $\zeta_{\boldsymbol{k}_{L}}$ does not stop evolving in time in the limit $\boldsymbol{k}_{L} \rightarrow 0$ as in models with multi light fields and also in nonattractor models. ${ }^{8}$ In fact, the condition (2.39) states that performing the inhomogeneous dilatation transformation that induces the time-independent shift of $\zeta_{\boldsymbol{k}_{L}}$, is equivalent to shifting (the eigen value of) $\zeta_{\boldsymbol{k}_{L}}$ at the evaluation time, which implies that the inserted soft mode should be dominated by a constant contribution.

Now, we show that the condition (2.39) can be indeed understood as the locality condition. For this purpose, we consider a set of eigenstates for the Fourier mode $\zeta_{\boldsymbol{k}_{L}}$ which satisfies $\zeta_{\boldsymbol{k}_{L}}\left|\zeta_{L}^{c}\right\rangle=\zeta_{\boldsymbol{k}_{L}}^{c}\left|\zeta_{L}^{c}\right\rangle$ instead of the eigenstates for the collective mode $\tilde{\zeta}_{\boldsymbol{k}_{L}}$. Using the complete set of $\left|\zeta_{L}^{c}\right\rangle$, we decompose the quantum state $|\Psi\rangle$ as

$$
|\Psi\rangle=\int \mathcal{D} \zeta_{L}\left|\psi\left(\zeta_{L}^{c}\right)\right|\left|\zeta_{L}^{c}\right\rangle|\Psi\rangle \zeta_{L}^{c}
$$

where $\mathcal{D} \zeta_{L}$ represents the functional integral over all soft modes. The extension of eq. (2.39) to the case of continuous modes will be

$$
i Q_{\zeta}^{W}\left(\boldsymbol{k}_{L}\right)|\Psi\rangle_{\zeta_{L}^{c}}=s \hat{W}\left(-\boldsymbol{k}_{L}\right) \frac{\delta}{\delta \zeta_{-\boldsymbol{k}_{L}}^{c}}|\Psi\rangle_{\zeta_{L}^{c}}
$$

\footnotetext{
${ }^{8}$ According to our understanding, in solid inflation [45], the anisotropic pressure plays the role of an additional degree of freedom, leading to the non-conservation of $\zeta$ and also the violation of the consistency relation. This is consistent with the statement shown in ref. [46] that the consistency relation can be recovered by taking angular average over the long mode.
} 
Using this relation, we obtain

$$
i Q_{\zeta}^{W}(\boldsymbol{x})|\Psi\rangle_{\tilde{\zeta}_{L}^{c}}=s \int \frac{d^{3} \boldsymbol{k}_{L}}{(2 \pi)^{3}} e^{-i \boldsymbol{k}_{L} \cdot \boldsymbol{x}} \hat{W}\left(\boldsymbol{k}_{L}\right) \frac{\delta}{\delta \zeta_{\boldsymbol{k}_{L}}}|\Psi\rangle_{\zeta_{L}^{c}}=s \frac{\delta}{\delta \zeta_{L}(\boldsymbol{x})}|\Psi\rangle_{\zeta_{L}^{c}},
$$

where

$$
\zeta_{L}(\boldsymbol{x}) \equiv \int \frac{d^{3} \boldsymbol{k}_{L}}{(2 \pi)^{3}} \hat{W}\left(\boldsymbol{k}_{L}\right) e^{i \boldsymbol{k}_{L} \cdot \boldsymbol{x}} \zeta_{\boldsymbol{k}_{L}}
$$

is the coarse grained field corresponding to the degrees of freedom of soft modes. Let us imagine a set of the separate universes whose sizes are of $\mathcal{O}(L)$. The operator $Q_{\zeta}^{W}(\boldsymbol{x})$ induces the dilatation only within the separate universe centered at $\boldsymbol{x}$. The condition (2.52) states that the impact of the soft mode on the quantum state of short modes is limited only to the influence which is equivalent to the inhomogeneous dilatation in the separate universe.

Notice that since (the amplitude of) the wave function is not flat in the direction of the inhomogeneous mode of $\zeta$, i.e., $\partial\left|\psi\left(\tilde{\zeta}_{\boldsymbol{k}_{L}}\right)\right| / \partial \tilde{\zeta}_{\boldsymbol{k}_{L}} \neq 0$, as seen in eq. (2.44), the quantum state $|\Psi\rangle$ does not remain invariant under the inhomogeneous dilatation $Q_{\zeta}^{W}\left(\boldsymbol{k}_{L}\right)$, i.e.,

$$
Q_{\zeta}^{W}\left(\boldsymbol{k}_{L}\right)|\Psi\rangle \neq 0
$$

As shown in eq. (2.45), operating the generator of the inhomogeneous dilatation inserts the soft mode $\tilde{\zeta}_{\boldsymbol{k}_{L}}$ with $\boldsymbol{k}_{L} \neq 0$, which changes the quantum state, even if the state is invariant under the homogeneous dilatation. As will be discussed in section 4, because of that, choosing a quantum state which is invariant under the dilatation is not enough to guarantee the IR regularity. Let us emphasize that there is no spontaneous symmetry breaking for the large gauge transformations: the symmetry under the dilatation, generated by $Q_{\zeta}$, is preserved also after the quantization, while the inhomogeneous dilatation, generated by $Q_{\zeta}^{W}$, is not a symmetry of the classical action.

Although we assumed that the wave function of the soft mode, $\left|\psi\left(\tilde{\zeta}_{\boldsymbol{k}_{L}}^{c}\right)\right|$, is given by the Gaussian distribution, the non-linear interactions of the hard modes are fully kept. Therefore, the consistency relation thus derived can apply to a much more general setup compared to the original one by Maldacena in ref. [14]. For instance, the wavelengths of the hard modes can be arbitrary as far as $k_{S} L \simeq k_{S} / k_{L} \gg 1$, i.e., they are not necessarily in super Hubble scales. In addition, obviously the same argument as above can apply, even if the time coordinates of the short modes $\zeta_{\boldsymbol{k}_{S i}}\left(t_{i}\right)$ with $i=1, \cdots, n$ are different among them.

Because of the Gaussian approximation for $\psi\left(\tilde{\zeta}_{\boldsymbol{k}_{L}}^{c}\right)$, the consistency relation derived here do not contain non-linear interactions of the soft modes. Notice that since there is no approximation for the soft modes in the locality condition (2.39) or (2.52), when we include the non-linear contributions of the soft modes, the same condition leads to the consistency relation. ${ }^{9}$ However, the non-linear interactions of the soft modes in general yield the IR divergences through their radiative corrections. Therefore, this extension requires a more careful consideration. This issue will be discussed in section 4 .

\footnotetext{
${ }^{9}$ When we include the non-linear contributions of the soft modes, the dilatation also changes the argument of $\zeta$ and the transformation of $\zeta_{\boldsymbol{k}_{L}}$ under the dilatation is not the simple shift. In order not to change the argument, we need to introduce the window function in a physical distance such as the geodesic distance.
} 


\section{Massive particles with arbitrary spins}

In this section, we consider an influence of heavy fields with arbitrary spins, which interact with the inflaton directly or indirectly through the gravitational interaction. The argument in this section is a generalization of the one in ref. [19], which showed the consistency relation for a heavy scalar field and the conservation of $\zeta$, taking into account radiative corrections of the heavy scalar field. In the previous section, we derived the condition for the consistency relation, using the generator(s) of the inhomogeneous dilatation. This argument can be extended to the cases with the radiative corrections of massive non-zero spin fields straightforwardly. In this section and in appendix B, to keep generality, we consider a $(d+1)$-dimensional spacetime.

\subsection{Setup of the problem}

In this section, we consider a heavy field whose mass is $M_{S}$ and spin is $S \geq 0$, including higher spin fields. We only consider the mass range where there is no instability [21, 47], e.g., $M_{2} \geq 2 H$ for a spin 2 field. For our purpose, we do not need to specify the detail of the interaction for the interacting system with the inflaton and the massive fields. We simply express the action as

$$
S[\delta g, \chi]=S_{a d}[\delta g]+S_{\chi}[\delta g, \chi]
$$

with

$$
S_{\chi}[\delta g, \chi] \equiv \sum_{\alpha} \int d t d^{d} \boldsymbol{x} a^{d} e^{d \zeta(x)} f^{\left\{i_{\alpha}\right\}}(\phi, \delta g) O_{\left\{i_{\alpha}\right\}}(x),
$$

where $\chi$ and $O_{\left\{i_{\alpha}\right\}}$ denote a set of heavy fields $\chi^{I}$ with $I=1,2, \cdots$ and a composite operator of $\chi$, respectively, and $\delta g$ denotes the set of the metric perturbations, $N, N_{i}, \zeta$, and $\gamma_{i j}$. Here, the action $S_{a d}[\delta g]$ only includes the metric perturbations in the unitary gauge defined by the condition $\delta \phi=0$ and it is identical to the action in single field models of inflation.

The heavy fields and the inflaton $\phi$ also can interact directly. For the present purpose, we do not need to specify the composite operators $O_{\left\{i_{\alpha}\right\}}$. We only need to specify their scaling dimensions $\Delta_{\alpha}$, i.e., they transform as

$$
O_{\left\{i_{\alpha}\right\}}^{s}\left(t, \boldsymbol{x}_{s}\right)=e^{-\Delta_{\alpha} s} O_{\left\{i_{\alpha}\right\}}(t, \boldsymbol{x})
$$

under the dilatation transformation $\boldsymbol{x} \rightarrow \boldsymbol{x}_{s}=e^{s} \boldsymbol{x}$, where $i_{\alpha}$ denotes tensor indices. While the heavy fields can be a fermion with a half integer spin, we assume that $\delta g$ interacts with $\chi$ only through the composite operators which transform as tensors (with an integer spin) under coordinate transformations. A composite operator with $n$ tensor (lower) indices has a scaling dimension $n$, e.g., $\Delta=0$ for a scalar composite operator and $\Delta=1$ for a vector one.

\subsection{Soft theorem for heavy fields with non-zero spins}

In this subsection, we derive the consistency relation or the soft theorem in the presence of the heavy fields, extending the discussion in the previous section. In the previous section, expanding the quantum state $|\Psi\rangle$ as in eq. (2.21), we derived the conditions (2.28) 
and (2.29) by requesting the invariance of the quantum state under the dilatation. Here, repeating the same argument except that now the quantum state $|\Psi\rangle$ also includes the heavy fields in addition to the inhomogeneous modes of $\zeta$, we obtain the same conditions as eqs. (2.28) and (2.29) from the dilatation invariance of $|\Psi\rangle$. Since $|\Psi\rangle_{\bar{\zeta}^{c}}$ also includes the degrees of freedom for the heavy fields, the condition (2.29) restricts how both of the inhomogeneous modes of $\zeta$ and the heavy fields should respond to the dilatation transformation. Similarly, when the condition (2.29) can be extrapolated to the inhomogeneous dilatation with the fall-off at the spatial infinity by replacing the homogeneous mode $\bar{\zeta}^{c}$ in (2.29) with the soft mode $\tilde{\zeta}_{\boldsymbol{k}_{L}}$, i.e., when the locality condition (2.39) is fulfilled also in the interacting system with the inflaton and the heavy fields, we can derive the soft theorem, which describe the influence of the soft mode.

Recall that the consistency relation can be derived by evaluating the change of the quantum state $|\Psi\rangle$ and the change of the operators for the heavy fields. In order to derive the soft theorem for the heavy fields, we consider

$$
\left\langle\Psi\left|\left[i Q_{\zeta}^{W}\left(\boldsymbol{k}_{L}\right), O_{\left\{i_{\alpha_{1}}\right\} \boldsymbol{k}_{S 1}}\left(t_{1}\right) \cdots O_{\left\{i_{\alpha_{n}}\right\} \boldsymbol{k}_{S n}}\left(t_{n}\right)\right]\right| \Psi\right\rangle,
$$

where $O_{\left\{i_{\alpha}\right\} \boldsymbol{k}_{S}}(t)$ denotes the Fourier mode of $O_{\left\{i_{\alpha}\right\}}(x)$ with $k_{L} / k_{S} \ll 1$. Repeating the same argument and taking the limit $L_{c} \rightarrow \infty$, we find that the condition (2.39) implies eq. (2.45). Then, using eq. (2.45), we can compute the change of the quantum state under the inhomogeneous dilatation as

$$
\begin{aligned}
& \left\langle\Psi\left|\left[i Q_{\zeta}^{W}\left(\boldsymbol{k}_{L}\right), O_{\left\{i_{\alpha_{1}}\right\} \boldsymbol{k}_{S 1}}\left(t_{1}\right) \cdots O_{\left\{i_{\alpha_{n}}\right\} \boldsymbol{k}_{S n}}\left(t_{n}\right)\right]\right| \Psi\right\rangle \\
& \stackrel{\text { s.l. }}{\approx}-\frac{s_{-\boldsymbol{k}_{L}}}{P_{\zeta}\left(k_{L}\right)}\left\langle\Psi\left|\zeta_{\boldsymbol{k}_{L}} O_{\left\{i_{\alpha_{1}}\right\} \boldsymbol{k}_{S 1}}\left(t_{1}\right) \cdots O_{\left\{i_{\alpha_{n}}\right\} \boldsymbol{k}_{S n}}\left(t_{n}\right)\right| \Psi\right\rangle .
\end{aligned}
$$

We equate this expression with the one obtained by computing the change of the composite operators $O_{\left\{i_{\alpha}\right\} k}$ under the (inhomogeneous) dilatation. Since $O_{\left\{i_{\alpha}\right\} k}$ transforms as in eq. (3.3), we obtain

$$
\left[i Q_{\zeta}^{W}\left(\boldsymbol{k}_{L}\right), O_{\left\{i_{\alpha_{1}}\right\} \boldsymbol{k}_{S}}\right]=s_{-\boldsymbol{k}_{L}}\left(\frac{\partial}{\partial \boldsymbol{k}_{S}} \boldsymbol{k}_{S}-\Delta_{\alpha}\right) O_{\left\{i_{\alpha_{1}}\right\} \boldsymbol{k}_{S}} .
$$

Likewise in the discussion for the single field case, whether the dilatation parameter is homogeneous or inhomogeneous does not affect the transformation of the short modes. Using this expression, we obtain

$$
\begin{aligned}
& \left\langle\Psi\left|\left[i Q_{\zeta}^{W}\left(\boldsymbol{k}_{L}\right), O_{\left\{i_{\alpha_{1}}\right\} \boldsymbol{k}_{S 1}}\left(t_{1}\right) \cdots O_{\left\{i_{\alpha_{n}}\right\} \boldsymbol{k}_{S n}}\left(t_{n}\right)\right]\right| \Psi\right\rangle \\
& \quad \stackrel{\text { s.l. }}{\approx} s_{-\boldsymbol{k}_{L}} \sum_{i=1}^{n}\left(\partial_{\boldsymbol{k}_{S i}} \boldsymbol{k}_{S i}-\Delta_{\alpha_{i}}\right)\left\langle\Psi\left|O_{\left\{i_{\alpha_{1}}\right\} \boldsymbol{k}_{S 1}}\left(t_{1}\right) \cdots O_{\left\{i_{\alpha_{n}}\right\} \boldsymbol{k}_{S n}}\left(t_{n}\right)\right| \Psi\right\rangle .
\end{aligned}
$$

Equating these two expressions and sending $L_{c}$ to the infinity, we obtain the consistency relation for the heavy fields as

$$
\begin{aligned}
& \lim _{k_{L} \rightarrow 0} \frac{\left\langle\Psi\left|\zeta_{\boldsymbol{k}_{L}} O_{\left\{i_{\alpha_{1}}\right\} \boldsymbol{k}_{S 1}}\left(t_{1}\right) \cdots O_{\left\{i_{\alpha_{n}}\right\} \boldsymbol{k}_{S n}}\left(t_{n}\right)\right| \Psi\right\rangle^{\prime}}{P_{\zeta}\left(k_{L}\right)} \\
& \stackrel{\text { s.l. }}{\approx}-\left(\sum_{i=2}^{n} \boldsymbol{k}_{S i} \cdot \frac{\partial}{\partial \boldsymbol{k}_{S i}}+d(n-1)-\Delta\right)\left\langle\Psi\left|O_{\left\{i_{\alpha_{1}}\right\} \boldsymbol{k}_{S 1}}\left(t_{1}\right) \cdots O_{\left\{i_{\alpha_{n}}\right\} \boldsymbol{k}_{S n}}\left(t_{n}\right)\right| \Psi\right\rangle^{\prime},
\end{aligned}
$$


where we defined $\Delta \equiv \sum_{i=1}^{n} \Delta_{\alpha_{i}}$. We put a prime to denote the correlation functions without the multiplicative factor $(2 \pi)^{d}$ and the delta function which expresses the momentum conservation.

\subsection{Effective action}

In order to show that the radiative corrections of the heavy fields do not induce any time evolution of $\zeta$ at large scales when the condition (2.39) is satisfied, we compute the effective action for $\zeta$ by integrating out the heavy fields $\chi$ in the closed time path (or the in-in) formalism. In particular, the contributions of the heavy fields $\chi$ are described by the Feynman and Vernon's influence functional [48, 49]. In this subsection, we briefly summarize the way to calculate the influence functional and the effective action. We will see that now the argument to show the conservation proceeds almost in parallel to the one for the heavy scalar field, discussed in ref. [19].

\subsubsection{Influence functional}

Performing the path integral along the closed time path, the $n$-point function of the curvature perturbation $\zeta$ is given by

$$
\begin{aligned}
& \left\langle\Psi\left|T \zeta\left(x_{1}\right) \cdots \zeta\left(x_{n}\right)\right| \Psi\right\rangle \\
& =\frac{\int D \delta g_{+}^{d y} \int D \chi_{+} \int D \delta g_{-}^{d y} \int D \chi_{-} \zeta_{+}\left(x_{1}\right) \cdots \zeta_{+}\left(x_{n}\right) e^{i S\left[\delta g_{+}, \chi_{+}\right]-i S\left[\delta g_{-}, \chi_{-}\right]}}{\int D \delta g_{+}^{d y} \int D \chi_{+} \int D \delta g_{-}^{d y} \int D \chi_{-} e^{i S\left[\delta g_{+}, \chi_{+}\right]-i S\left[\delta g_{-}, \chi_{-}\right]}},
\end{aligned}
$$

where we double the fields: $\delta g_{+}$and $\chi_{+}$denote the fields defined along the path from the past infinity to the time $t$ and $\delta g_{-}$and $\chi_{-}$denote the fields integrated from the time $t$ to the past infinity. Since $N$ and $N_{i}$ are the Lagrange multiplies, which are eliminated by solving the constraint equations, we perform the path integral only regarding the dynamical degrees of freedom $\delta g^{d y} \equiv\left(\zeta, \gamma_{i j}\right)$ and $\chi$. An insertion of $\delta g_{+}(x)$ into the path integral in the numerator as above gives a correlation function in the time ordering, expressed by $T$, while an insertion of $\delta g_{-}(x)$ gives a correlator in the anti-time ordering, expressed by $\bar{T}$.

Separating the part which describes the radiative corrections of the heavy fields as

$$
i S_{\mathrm{eff}}\left[\delta g_{+}, \delta g_{-}\right] \equiv \ln \left[\int D \chi_{+} \int D \chi_{-} e^{i S\left[\delta g_{+}, \chi_{+}\right]-i S\left[\delta g_{-}, \chi_{-}\right]}\right],
$$

we can express the $n$-point function for $\zeta$ superficially as if there are only the metric perturbations and the inflaton as

$$
\left\langle\Psi\left|T \zeta\left(x_{1}\right) \cdots \zeta\left(x_{n}\right)\right| \Psi\right\rangle=\frac{\int D \delta g_{+}^{d y} \int D \delta g_{-}^{d y} \zeta_{+}\left(x_{1}\right) \cdots \zeta_{+}\left(x_{n}\right) e^{i S_{\mathrm{eff}}\left[\delta g_{+}, \delta g_{-}\right]}}{\int D \delta g_{+}^{d y} \int D \delta g_{-}^{d y} e^{i S_{\mathrm{eff}}\left[\delta g_{+}, \delta g_{-}\right]}} .
$$

The effective action is recast into

$$
S_{\text {eff }}\left[\delta g_{+}, \delta g_{-}\right]=S_{a d}\left[\delta g_{+}\right]-S_{a d}\left[\delta g_{-}\right]+S_{\text {eff }}^{\prime}\left[\delta g_{+}, \delta g_{-}\right],
$$

where $S_{\text {eff }}^{\prime}$ is the so-called influence functional, given by

$$
i S_{\mathrm{eff}}^{\prime}\left[\delta g_{+}, \delta g_{-}\right] \equiv \ln \left[\int D \chi_{+} \int D \chi_{-} e^{i S_{\chi}\left[\delta g_{+}, \chi_{+}\right]-i S_{\chi}\left[\delta g_{-}, \chi_{-}\right]}\right]
$$


where we factorized $S_{a d}\left[\delta g_{ \pm}\right]$which commutes with the path integral over $\chi_{ \pm}$. Here, we only consider the correlation functions for $\zeta$, but the effective action $S_{\text {eff }}\left[\delta g_{+}, \delta g_{-}\right]$describes the evolution of both $\zeta$ and $\gamma_{i j}$ affected by the quantum fluctuations of the heavy fields $\chi$. For our later use, we introduce the correlation functions of $\chi$ computed in the absence of the metric perturbations as

$$
\left\langle O\left[\chi_{+}, \chi_{-}\right]\right\rangle_{ \pm} \equiv \frac{\int D \chi_{+} \int D \chi_{-} O\left[\chi_{+}, \chi_{-}\right] e^{i S_{\chi}\left[0, \chi_{+}\right]-i S_{\chi}\left[0, \chi_{-}\right]}}{\int D \chi_{+} \int D \chi_{-} e^{i S_{\chi}\left[0, \chi_{+}\right]-i S_{\chi}\left[0, \chi_{-}\right]}}
$$

Expanding $S_{\text {eff }}^{\prime}$ in terms of the metric perturbations $\delta g=\left(\delta N, N_{i}, \zeta, \gamma_{i j}\right)$, we obtain

$$
i S_{\mathrm{eff}}^{\prime}\left[\delta g_{+}, \delta g_{-}\right] \equiv \sum_{n=0}^{\infty} i S_{\mathrm{eff}(n)}^{\prime}\left[\delta g_{+}, \delta g_{-}\right]
$$

where $S_{\text {eff }(n)}^{\prime}$ denotes the terms which include $n \delta g_{\alpha}$ s, given by

$$
\begin{aligned}
i S_{\mathrm{eff}(n)}^{\prime}\left[\delta g_{+}, \delta g_{-}\right]= & \frac{1}{n !} \sum_{a_{1}= \pm} \cdots \sum_{a_{n}= \pm} \int d^{d+1} x_{1} \cdots \int d^{d+1} x_{n} \\
& \times \delta g_{a_{1}}\left(x_{1}\right) \cdots \delta g_{a_{n}}\left(x_{n}\right) W_{\delta g_{a_{1}} \cdots \delta g_{a_{n}}}^{(n)}\left(x_{1}, \cdots, x_{n}\right)
\end{aligned}
$$

with the non-local interaction vertices induced by the heavy fields:

$$
\left.W_{\delta g_{a_{1}} \cdots \delta g_{a_{n}}}^{(n)}\left(x_{1}, \cdots, x_{n}\right) \equiv \frac{\delta^{n} i S_{\mathrm{eff}}^{\prime}\left[\delta g_{+}, \delta g_{-}\right]}{\delta g_{a_{1}}\left(x_{1}\right) \cdots \delta g_{a_{n}}\left(x_{n}\right)}\right|_{\delta g_{ \pm}=0} .
$$

In eq. (3.16), each $\delta g_{a_{m}}$ with $m=1, \cdots, n$ should add up all the metric perturbations $\delta N_{a_{m}}, N_{i, a_{m}}, \zeta_{a_{m}}, \gamma_{i j a_{m}}$. Here and hereafter, for notational brevity, we omit the summation symbol over $\delta g$ unless necessary. Inserting eq. (3.13) into eq. (3.17), we can express the nonlocal interaction vertices by using the correlators for $\chi$. These expressions are summarized in appendix B. Once we expand the effective action as in eq. (3.16), the shift symmetry is lost at each order in perturbation about $\delta g$. In the following, we will show that we can rewrite the effective action in such a way that the shift symmetry is manifestly preserved by using the consistency relation.

\subsubsection{Soft theorem and effective action}

In order to show the conservation of $\zeta$ in the presence of the radiative corrections of the heavy fields, here we rewrite the consistency relation (3.8). As was mentioned in the previous section, the time coordinates of the hard modes can be different among different composite operators. Taking an appropriate ordering of the composite operators, i.e., we can put the index $a_{i}= \pm$ on each composite operator $O_{\left\{i_{i}\right\} \boldsymbol{k}_{S i}}$ in the consistency relation (3.8). In the following, we use the prescription introduced in the previous subsection (see also appendix B). In particular, all the correlation functions should be understood as being computed in the path ordering of the closed time path with the distinction of \pm . 
Employing the Gaussian approximation for the soft mode of $\zeta$ again, we can compute the correlation function in the first line of eq. (3.8) as

$$
\begin{aligned}
& \left\langle\Psi\left|\zeta_{\boldsymbol{k}_{L}} O_{\left\{i_{\alpha_{1}}\right\} \boldsymbol{k}_{S 1}}^{a_{1}}\left(t_{1}\right) \cdots O_{\left\{i_{\alpha_{n}}\right\} \boldsymbol{k}_{S n}}^{a_{n}}\left(t_{n}\right)\right| \Psi\right\rangle \\
& \stackrel{\stackrel{\mathrm{sl}}{\approx}}{\approx}-i\left\langle\Psi, 0_{\zeta}\left|S_{\chi}^{\mathrm{int}-} \zeta_{\boldsymbol{k}_{L}} O_{\left\{i_{\alpha_{1}}\right\} \boldsymbol{k}_{S 1}}^{a_{1}}\left(t_{1}\right) \cdots O_{\left\{i_{\alpha_{n}}\right\} \boldsymbol{k}_{S n}}^{a_{n}}\left(t_{n}\right)\right| \Psi, 0_{\zeta}\right\rangle \\
& \quad+i\left\langle\Psi, 0_{\zeta}\left|\zeta_{\boldsymbol{k}_{L}} O_{\left\{i_{\alpha_{1}}\right\} \boldsymbol{k}_{S 1}}^{a_{1}}\left(t_{1}\right) \cdots O_{\left\{i_{\alpha_{n}}\right\} \boldsymbol{k}_{S n}}^{a_{n}}\left(t_{n}\right) S_{\chi}^{\mathrm{int}+}\right| \Psi, 0_{\zeta}\right\rangle,
\end{aligned}
$$

where $\left|\Psi, 0_{\zeta}\right\rangle$ denotes the quantum state $|\Psi\rangle$ with $\zeta$ being in non-interacting vacuum. Here, $S_{\chi}^{\text {int }}$ denotes a set of the interaction vertexes which include only one $\zeta$ without derivative and the massive fields $\chi$ and is given by

$$
S_{\chi}^{\text {int }}=\left.\int d t d^{d} \boldsymbol{x} \zeta(x) \frac{\delta S_{\chi}}{\delta \zeta(x)}\right|_{\zeta=0}=\left.\int d t \int \frac{d^{d} \boldsymbol{k}}{(2 \pi)^{d}} \zeta_{\boldsymbol{k}} \frac{\delta S_{\chi}}{\delta \zeta}\right|_{\zeta=0}(t,-\boldsymbol{k})
$$

with

$$
\left.\left.\frac{\delta S_{\chi}}{\delta \zeta}\right|_{\zeta=0}(t, \boldsymbol{k}) \equiv \int d^{d} \boldsymbol{x} e^{-i \boldsymbol{k} \cdot \boldsymbol{x}} \frac{\delta S_{\chi}}{\delta \zeta(x)}\right|_{\zeta=0}
$$

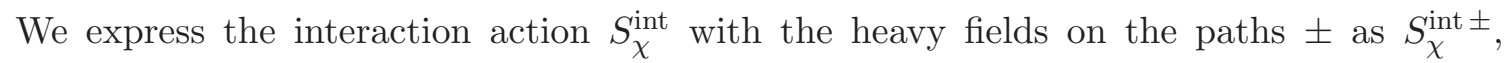
respectively. Factoring out the power spectrum of $\zeta$, we obtain

$$
\begin{aligned}
& \frac{\left\langle\Psi\left|\zeta_{\boldsymbol{k}_{L}} O_{\left\{i_{\alpha_{1}}\right\} \boldsymbol{k}_{S 1}}^{a_{1}}\left(t_{1}\right) \cdots O_{\left\{i_{n}\right\} \boldsymbol{k}_{S n}}^{a_{n}}\left(t_{n}\right)\right| \Psi\right\rangle}{P_{\zeta}\left(k_{L}\right)} \\
& \stackrel{\text { s.l. }}{\approx}-i \int d t\left\langle\Psi, 0_{\zeta}\left|\frac{\delta S_{\chi}^{-}}{\delta \zeta}\right|_{\zeta=0}\left(t, \boldsymbol{k}_{L}\right) O_{\left\{i_{\alpha_{1}}\right\} \boldsymbol{k}_{S 1}}^{a_{1}}\left(t_{1}\right) \cdots O_{\left\{i_{n}\right\} \boldsymbol{k}_{S n}}^{a_{n}}\left(t_{n}\right) \mid \Psi, 0_{\zeta}\right\rangle \\
& \quad+i \int d t\left\langle\Psi, 0_{\zeta}\left|O_{\left\{i_{\alpha_{1}}\right\} \boldsymbol{k}_{S 1}}^{a_{1}}\left(t_{1}\right) \cdots O_{\left\{i_{\alpha_{n}}\right\} \boldsymbol{k}_{S n}}^{a_{n}}\left(t_{n}\right) \frac{\delta S_{\chi}^{+}}{\delta \zeta}\right|_{\zeta=0}\left(t, \boldsymbol{k}_{L}\right) \mid \Psi, 0_{\zeta}\right\rangle,
\end{aligned}
$$

where the power spectrum of $\zeta$ is canceled between the numerator and the denominator. In the first line of eq. (3.21), we omitted the time coordinate of the soft mode $\zeta_{\boldsymbol{k}_{L}}$, since it should be constant in time to satisfy the condition (2.39). (If one wants to specify the time coordinate of $\zeta_{\boldsymbol{k}_{L}}$, we can place it along the closed time path at the end of inflation where $\zeta_{\boldsymbol{k}_{L}}^{+}=\zeta_{\boldsymbol{k}_{L}}^{-}$.) Expressing the composite operators in the position space, we obtain

$$
\begin{aligned}
& \sum_{i=1}^{n}\left\{\partial_{\boldsymbol{x}_{i}} \boldsymbol{x}_{i}-\left(d-\Delta_{i}\right)\right\}\left\langle\Psi, 0_{\zeta}\left|O_{\left\{i_{\alpha_{1}}\right\}}^{a_{1}}\left(x_{1}\right) \cdots O_{\left\{i_{\alpha_{n}}\right\}}^{a_{n}}\left(x_{n}\right)\right| \Psi, 0_{\zeta}\right\rangle \\
& \stackrel{\text { s.l. }}{\approx}-i \int d t\left\langle\Psi, 0_{\zeta}\left|\frac{\delta S_{\chi}^{-}}{\delta \zeta}\right|_{\zeta=0}\left(t, \boldsymbol{k}_{L}\right) O_{\left\{i_{\alpha_{1}}\right\}}^{a_{1}}\left(x_{1}\right) \cdots O_{\left\{i_{\alpha_{n}}\right\}}^{a_{n}}\left(x_{n}\right) \mid \Psi, 0_{\zeta}\right\rangle \\
& \quad+i \int d t\left\langle\Psi, 0_{\zeta}\left|O_{\left\{i_{\alpha_{1}}\right\}}^{a_{1}}\left(x_{1}\right) \cdots O_{\left\{i_{\alpha_{n}}\right\}}^{a_{n}}\left(x_{n}\right) \frac{\delta S_{\chi}^{+}}{\delta \zeta}\right|_{\zeta=0}\left(t, \boldsymbol{k}_{L}\right) \mid \Psi, 0_{\zeta}\right\rangle
\end{aligned}
$$

Since we neglected the higher order contributions of the soft modes using the approximation of $\stackrel{\text { s.l. }}{\approx}$, we replaced the quantum state $|\Psi\rangle$ in the first line with $\left|\Psi, 0_{\zeta}\right\rangle$. 


\subsection{Conservation of $\zeta$}

For the purpose of showing the conservation of $\zeta$, let us introduce

$$
\delta \hat{g}(x)=\left\{N(x), e^{-\zeta(x)} N_{i}(x), \dot{\zeta}(x), e^{-\zeta(x)} \partial_{x} \zeta(x), e^{-\zeta(x)} \partial_{x}, e^{-2 \zeta(x)} \gamma_{i j}(x)\right\}
$$

Since the metric perturbations transform as

$$
\begin{aligned}
N_{s}\left(t, \boldsymbol{x}_{s}\right) & =N(t, \boldsymbol{x}), & e^{s} N_{i, s}\left(t, \boldsymbol{x}_{s}\right) & =N_{i}(t, \boldsymbol{x}), \\
\zeta_{s}\left(t, \boldsymbol{x}_{s}\right)+s & =\zeta(t, \boldsymbol{x}), & e^{2 s} \gamma_{i j s}\left(t, \boldsymbol{x}_{s}\right) & =\gamma_{i j}(t,, \boldsymbol{x})
\end{aligned}
$$

under the dilatation transformation, we can easily see that $\delta \hat{g}(x)$ transform as a scalar under the dilatation.

Taking into account that the composite operator $O_{\left\{i_{\alpha}\right\}}$ transforms as given in eq. (3.3), we also can construct a scalar operator for $O_{\left\{i_{\alpha}\right\}}$ as $e^{-\Delta_{\alpha} \zeta(x)} O_{\left\{i_{\alpha}\right\}}(x)$. Here, introducing

$$
\hat{f}^{\left\{i_{\alpha}\right\}}(\phi, \delta \hat{g}) \equiv e^{\Delta_{\alpha} \zeta} f^{\left\{i_{\alpha}\right\}}(\phi, \delta g)
$$

for each $f^{\left\{i_{\alpha}\right\}}$ in the action (3.2), we rewrite the action for the heavy fields as

$$
S_{\chi}[\delta g, \chi] \equiv \sum_{a} \int d t d^{d} \boldsymbol{x} a^{d} e^{d \zeta(x)} \hat{f}^{\left\{i_{\alpha}\right\}}(\phi, \delta \hat{g}) e^{-\Delta_{\alpha} \zeta(x)} O_{\left\{i_{\alpha}\right\}}(x)
$$

Since $\hat{f}^{\left\{i_{a}\right\}}$ transforms as a scalar under the dilatation, the metric perturbations included in $\hat{f}^{\left\{i_{a}\right\}}$ can be expressed only in terms of $\delta \hat{g}(x)$, which also transform as a scalar.

Taking the first and the second variations of $S_{\chi}$ with respect to $\zeta$, we obtain

$$
\begin{aligned}
\left.\frac{\delta S_{\chi}}{\delta \zeta(x)}\right|_{\delta g=0} & =\sum_{\alpha} a^{d}(t)\left(d-\Delta_{\alpha}\right) f^{\left\{i_{\alpha}\right\}} O_{\left\{i_{\alpha}\right\}}(x), \\
\left.\frac{\delta^{2} S_{\chi}}{\delta \zeta^{2}(x)}\right|_{\delta g=0} & =\sum_{\alpha} a^{d}(t)\left(d-\Delta_{\alpha}\right)^{2} f^{\left\{i_{\alpha}\right\}} O_{\left\{i_{\alpha}\right\}}(x) .
\end{aligned}
$$

Since we expressed the action as in eq. (3.27), the terms in which the derivative operates on $\hat{f}^{\left\{i_{\alpha}\right\}}$ vanish. Variations with respect to the other metric perturbations, $\delta N, e^{-\zeta} N_{i}, e^{-2 \zeta} \gamma_{i j}$, which are in the combination of $\delta \hat{g}$, yield

$$
\begin{aligned}
\left.\frac{\delta S_{\chi}}{\delta \hat{g}(x)}\right|_{\delta g=0} & =\left.\sum_{\alpha} a^{d}(t) \frac{\partial \hat{f}^{\left\{i_{\alpha}\right\}}}{\partial \hat{g}(x)}\right|_{\delta g=0} O_{\left\{i_{\alpha}\right\}}(x), \\
\left.\frac{\delta^{2} S_{\chi}}{\delta \hat{g}(x) \delta \zeta(x)}\right|_{\delta g=0} & =\left.\sum_{\alpha} a^{d}(t)\left(d-\Delta_{\alpha}\right) \frac{\partial \hat{f}^{\left\{i_{\alpha}\right\}}}{\partial \hat{g}(x)}\right|_{\delta g=0} O_{\left\{i_{\alpha}\right\}}(x),
\end{aligned}
$$

and so on. Multiplying $a^{d}(t) \partial \hat{f}^{\left\{i_{\alpha}\right\}} /\left.\partial g(x)\right|_{\delta g=0}$ on eq. (3.22) with $n=1$ and all the remaining metric perturbations set to 0 and taking summation over $\alpha$, we obtain

$$
\partial_{\boldsymbol{x}}\left\{\boldsymbol{x} W_{\delta g_{ \pm}}^{(1)}(x)\right\} \stackrel{\text { s.l. }}{\approx} \int d t_{y} \int d^{d} \boldsymbol{y} e^{-i \boldsymbol{k}_{L} \cdot \boldsymbol{y}}\left\{W_{\delta g_{ \pm} \zeta_{+}}^{(2)}(x, y)+W_{\delta g_{ \pm} \zeta_{-}}^{(2)}(x, y)\right\}
$$


for $\delta g=\zeta, \delta N, N_{i}, \gamma_{i j}$. Notice that the derivative with respect to $e^{-\zeta} N_{i}$ and the one with respect to $N_{i}$ give the same answer after setting $\delta g$ to 0 . The same story also follows for $\gamma_{i j}$. Here, we expressed $\delta S_{\chi} /\left.\delta \zeta\right|_{\zeta=0}\left(t, \boldsymbol{k}_{L}\right)$ in eq. (3.22) in the position space. In deriving eq. (3.22), we used the explicit forms of $W_{\delta g_{a_{1}} \delta \tilde{g}_{a_{2}}}^{(2)}\left(x_{1}, x_{2}\right)$, summarized in appendix B. Multiplying $\delta g_{ \pm}$on eq. (3.32) and integrating over $x^{\mu}$, we finally obtain the key formula to show the presence of the constant solution as

$$
\begin{aligned}
& \int d^{d+1} x\left\{\boldsymbol{x} \cdot \partial_{\boldsymbol{x}} \delta g_{ \pm}(x)\right\} W_{\delta g_{ \pm}}^{(1)}(x) \\
& \quad+\int d^{d+1} x \int d^{d+1} y e^{-i \boldsymbol{k}_{L} \cdot \boldsymbol{y}} \delta g_{ \pm}(x)\left\{W_{\delta g_{ \pm} \zeta_{+}}^{(2)}(x, y)+W_{\delta g_{ \pm} \zeta_{-}}^{(2)}(x, y)\right\} \stackrel{\text { s.l. }}{\approx} 0
\end{aligned}
$$

where we performed integration by parts. Meanwhile, multiplying $e^{-i \boldsymbol{k}_{L}^{\prime} \cdot \boldsymbol{x}}$ on eq. (3.32) and integrating over $x^{\mu}$, we obtain

$$
\int d^{d+1} x e^{-i \boldsymbol{k}_{L}^{\prime} \cdot \boldsymbol{x}} \int d^{d+1} y e^{-i \boldsymbol{k}_{L} \cdot \boldsymbol{y}}\left\{W_{\delta g_{ \pm} \zeta_{ \pm}}^{(2)}(x, y)+W_{\delta g_{ \pm} \zeta_{\mp}}^{(2)}(x, y)\right\}=0 .
$$

By adding the left hand side of eq. (3.33) multiplied by a constant parameter $-s$ and eq. (3.34) with $\delta g_{ \pm}=\zeta_{ \pm}$multiplied by $-s^{2} / 2$, the linear and the quadratic terms in the effective action can be given by

$$
\begin{aligned}
& i S_{\mathrm{eff}(1)}^{\prime}\left[\delta g_{+}, \delta g_{-}\right]+i S_{\mathrm{eff}(2)}^{\prime}\left[\delta g_{+}, \delta g_{-}\right] \\
& =\sum_{a= \pm} \int d^{d+1} x \delta g_{a, s}(x) W_{\delta g_{a}}^{(1)}(x) \\
& \quad+\frac{1}{2 !} \sum_{a_{1}, a_{2}= \pm} \int d^{d+1} x_{1} \int d^{d+1} x_{2} \delta g_{a_{1}, s}\left(x_{1}\right) \delta \tilde{g}_{a_{2}, s}\left(x_{2}\right) W_{\delta g_{a_{1}} \delta \tilde{g}_{a_{2}}}^{(2)}\left(x_{1}, x_{2}\right) \\
& \quad+\mathcal{O}\left(\delta g^{3}, \delta g^{2} s, \delta g s^{2}, s^{3}\right),
\end{aligned}
$$

where $\delta g_{s}$ denote the metric perturbations $\delta g$ after the inhomogeneous dilatation. For notational brevity, here we used the same notation as those for the global dilatation, given in eqs. (3.25) and (3.24). Here, each $\delta g_{i, a}(i=1,2)$ sums over $\delta N_{a, s}, N_{i, a, s}, \zeta_{a, s}$, and $\gamma_{i j, a, s}$. In deriving eq. (3.35), we used

$$
W_{\delta g_{a_{1}} \delta \tilde{g}_{a_{2}}}^{(2)}\left(x_{1}, x_{2}\right)=W_{\delta \tilde{g}_{a_{2}} \delta g_{a_{1}}}^{(2)}\left(x_{2}, x_{1}\right) .
$$

The first term in eq. (3.33) changes the argument of the metric perturbations in the linear term of the metric perturbations in eq. (3.35). We also changed the arguments of the quadratic terms, taking into account that the modification appears only in higher orders of perturbation. The tadpole contributions, which are the terms in the second line of eq. (3.35) should vanish by using the background equation of motion. (See ref. [19] for a discussion about the heavy scalar field.)

Equation (3.35) shows that with the use of the consistency relation, $\delta g_{\alpha}(x)$ in $S_{\text {eff }}^{\prime}$ can be replaced with $\delta g_{\alpha, s}(x)$. Since the rest of the effective action, $S_{a d}$, is simply the classical action for the single field model, it also should be invariant under this replacement. 
Therefore, when the locality condition (2.39) holds, the total effective action $S_{\text {eff }}$ preserves the invariance under the inhomogeneous dilatation with the suitable fall-off, i.e., under the change of $\delta g_{\alpha}$ to $\delta g_{s, \alpha}$. Since eqs. (3.33) and (3.34) also hold for the soft modes not only for the homogeneous mode with $\boldsymbol{k}=0$, we can shift $\zeta(x)$ by an inhomogeneous but timeindependent function instead of the homogeneous constant parameter. The invariance of the effective action, which also includes the radiative corrections from the non-zero spin massive fields, under the shift of the soft mode $\zeta_{\boldsymbol{k}_{L}}$ directly implies the existence of the constant solution for $\zeta .^{10}$

We assumed the locality condition (2.39) to derive the consistency relation, which was used to show the invariance under the replacement of $\zeta$ with $\zeta_{s}$ in the effective action. As was argued in section 2.3.2, the validity of eq. (2.39) requires the linear perturbation of the soft mode $\zeta_{\boldsymbol{k}_{L}}$ to be time independent. For that, the decaying mode of $\zeta_{\boldsymbol{k}_{L}}$ should die off sufficiently fast after the Hubble crossing. This happens when the background trajectory is on an attractor, e.g., when the heavy fields do not alter this nature of the background classical trajectory. As far as the radiative corrections of the heavy fields $\chi$ do not turn the "decaying" mode of $\zeta_{\boldsymbol{k}_{L}}$ into a growing mode, the existing constant solution should be the dominant solution of $\zeta$ in the large scale limit. This should be the case, when the radiative corrections of the heavy fields remain perturbative. In ref. [19], we listed the condition for the conservation of $\zeta$ in the presence of the radiative corrections of a massive (scalar) field as

- The radiative corrections of the heavy field are perturbatively small.

- The background trajectory is on an attractor.

- The quantum system preserves the dilatation invariance.

Now the second and third conditions are rephrased by the single condition:

- The locality condition (2.39) is satisfied.

This guarantees the presence of the constant solution also for the soft modes $\zeta_{\boldsymbol{k}_{L}}$ with $\boldsymbol{k}_{L} \neq 0$, not only for the homogeneous mode. The current argument also can apply to massive fields with arbitrary spins, including higher spin fields.

\section{IR divergences of inflationary correlators}

In the previous two sections, we showed that the locality condition (2.39) leads to the consistency relation and the conservation of $\zeta$. In this section, as another related subject, we show that the condition (2.39) also plays an important role for the cancellation of the IR divergent contributions.

\footnotetext{
${ }^{10}$ The lapse function and the shift vector, included in the effective action (3.35), can be eliminated by solving the Hamiltonian and momentum constraint equations and expressing them in terms of $\zeta_{s}$ as in the single field model [14]. Since the constraint equations for $\delta g_{s}$ are simply given by replacing $\delta g$ with $\delta g_{s}$ in the constraint equations for $\delta g$, the effective action obtained after eliminating these Lagrange multiplies obviously preserves the invariance under the replacement of $\zeta$ with $\zeta_{s}$.
} 


\subsection{Overview of IR divergence problem}

It is widely known that the loop corrections of a massless perturbation mode such as the curvature perturbation $\zeta$ can yield various IR enhancements. (See, e.g., refs. [50-58].) In this section, following ref. [59], we briefly summarize the IR enhancements. When we perform the perturbative expansion in terms of the interaction picture field $\zeta_{I}$, an interaction vertex which includes $\zeta_{I}$ without derivative can yield the radiative correction which is proportional to $\left\langle\zeta_{I}^{2}\right\rangle$. The super Hubble modes with $k \lesssim a H$ contribute to $\left\langle\zeta_{I}^{2}\right\rangle$ as $\int_{0}^{a H} d^{3} k / k^{3}$, yielding the logarithmic enhancement. We distinguish the divergent contribution due to the modes $0 \leq k \leq k_{c}$ (IRdiv) from the convergent but secularly growing one $(\propto \ln a)$ due to the modes $k_{c} \leq k \leq a H$ (IRsec). Here, $k_{c}$ denotes an IR cutoff. The IRsec, which cannot be removed by introducing the comoving IR cutoff, originates from the accumulation of the super Hubble modes. The accumulation of the super Hubble modes enhances the time integral at each interaction vertex (SG), introducing another secularly growing term proportional to $\ln a$ or increasing the power of $\ln a$ included in the integrand. These IR enhancements also can be introduced by the soft graviton.

\subsection{Cancellation of IR divergence and the locality condition}

In the series of papers $[39,40,44,59-64]$, we showed that the IR enhancements, i.e., IRdiv, IRsec, and SG, are due to the influences from the outside of the observable region. What we observe in cosmological measurements corresponds to a quantity evaluated in a completely fixed gauge. Fixing the gauge conditions eliminates the influence of the gauge degrees of freedom. However, it is not possible to determine the gauge condition outside the observable region, even if we completely specify the way of observation. Therefore, unless the causality is manifestly ensured as in the harmonic gauge, the degrees of freedom outside the observable region can affect the boundary conditions of the observable region. As was argued in refs. [39, 40], changing the boundary conditions corresponds to changing the spatial coordinates in the local observable region. Such spatial coordinate transformations are the large gauge transformations. We dubbed a variable which is independent of those boundary conditions of the local observable region as a genuine gauge invariant variable.

In refs. $[39,40,44,59,60,63,64]$, we showed that all the IR enhancements are cancelled out, when we calculate a correlation function of a genuine gauge invariant variable for a specific initial state such as the adiabatic vacuum. In ref. [63], we argued that whether the IR enhancements in the correlation function of the genuine gauge invariant operator disappear or not depends on the choice of the initial states. In this subsection, scrutinizing the condition on the quantum state to ensure the cancellation of the IR enhancements, we show that when the locality condition (2.39) is satisfied, the IRdiv, which comes from the momentum integral, is cancelled in the correlation functions of the invariant variable under the large gauge transformations. In the following, we denote a genuine gauge invariant variable as ${ }^{g} R(x)$ without specifying it. (One way to construct ${ }^{g} R(x)$ was discussed in refs. $[39,40]$. 
One important property of ${ }^{g} R$ is being constructed only by local quantities such that commute with the soft modes and remains invariant under the inhomogeneous dilatation, i.e.,

$$
{ }^{g} R(x)=e^{i Q_{\zeta}^{W}\left(\boldsymbol{k}_{L}\right) g} R(x) e^{-i Q_{\zeta}^{W}\left(\boldsymbol{k}_{L}\right)} .
$$

As in eq. (2.37), but focusing on a single soft mode, we expand the correlation function of ${ }^{g} R$ as

$$
\begin{aligned}
& \left\langle\Psi\left|{ }^{g} R\left(x_{1}\right) \ldots{ }^{g} R\left(x_{n}\right)\right| \Psi\right\rangle \\
& \quad=\int d \tilde{\zeta}_{\boldsymbol{k}_{L}}^{c}\left|\psi\left(\tilde{\zeta}_{\boldsymbol{k}_{L}}^{c}\right)\right|^{2}{ }_{\tilde{\zeta}_{\boldsymbol{k}_{L}}^{c}}\left\langle\Psi\left|{ }^{g} R\left(x_{1}\right) \ldots{ }^{g} R\left(x_{n}\right)\right| \Psi\right\rangle_{\tilde{\zeta}_{\boldsymbol{k}_{L}}^{c}}
\end{aligned}
$$

where we noted that the soft mode $\tilde{\zeta}_{\boldsymbol{k}_{L}}$ commutes with the genuine gauge invariant variable. When the "locality" condition holds, since ${ }^{g} R$ commutes with $Q_{\zeta}^{W}\left(\boldsymbol{k}_{L}\right)$ we obtain

$$
\begin{aligned}
0 & =\tilde{\zeta}_{\boldsymbol{k}_{L}^{c}}\left\langle\Psi\left|\left[i Q_{\zeta}^{W}\left(\boldsymbol{k}_{L}\right),{ }^{g} R\left(x_{1}\right) \cdots{ }^{g} R\left(x_{n}\right)\right]\right| \Psi\right\rangle_{\tilde{\boldsymbol{k}}_{L}^{c}} \\
& =\frac{\partial}{\partial \tilde{\zeta}_{\boldsymbol{k}_{L}}^{c}} \tilde{\zeta}_{\boldsymbol{k}_{L}}\left\langle\Psi\left|{ }^{g} R\left(x_{1}\right) \cdots{ }^{g} R\left(x_{n}\right)\right| \Psi\right\rangle_{\tilde{\zeta}_{\boldsymbol{k}_{L}}^{c}} .
\end{aligned}
$$

Since the correlator in eq. (4.3) is independent of the soft mode $\tilde{\zeta}_{\boldsymbol{k}_{L}}^{c}$, it commutes with the integral over $\tilde{\zeta}_{\boldsymbol{k}_{L}}^{c}$. Then, the divergent integral $\int d \tilde{\zeta}_{\boldsymbol{k}_{L}}^{c}\left|\psi\left(\tilde{\zeta}_{\boldsymbol{k}_{L}}^{c}\right)\right|^{2}$ in eq. (4.2) simply becomes the normalization factor, which should be canceled in computing connected diagrams. Here, we picked up a certain wavenumber $\boldsymbol{k}_{L}$. However, repeating the same procedure for the whole soft modes, we find that all the soft modes which correlate with the hard modes are canceled out. This cancellation yields a suppression of the soft modes which interact with the hard modes and ensures the absence of the IRdiv in the correlation function of ${ }^{g} R$. In this way, we find that while the quantum state $|\Psi\rangle$ is not invariant under the inhomogeneous dilatation, which inserts the soft mode $\zeta_{\boldsymbol{k}_{L}}$, the correlation function of ${ }^{g} R$ for the quantum state $|\Psi\rangle$ is insensitive to the insertion. In ref. [41], this cancellation of the correlation between the soft modes and the hard modes was presented by considering the squeezed bi-spectrum.

Here, let us further discuss the relation between the genuine gauge invariance and the absence of the IRdiv. Changing the boundary condition at the edge of the observable region, we can alter the spatial average of the curvature perturbation $\zeta[39,40,60]$. This can be expressed as the dilatation whose constant parameter $s$ is given by the spatial average of $\zeta$ in the observable region. This dilatation changes the constant part of all the modes with $k_{L} \lesssim 1 / L_{O}$, where $L_{O}$ is the size of the observable region, not only the homogeneous mode with $\boldsymbol{k}=0$. Therefore, the genuine gauge invariance requires being insensitive to the excitation of the constant soft modes $\zeta_{\boldsymbol{k}_{L}}$. As we argued in section 2.3.2 (see around eq. (2.54)), quantum states which satisfy the locality condition do not preserve the genuine gauge invariance in the sense that it is not insensitive to the insertion of the constant part of the soft modes $\zeta_{\boldsymbol{k}_{L}}$. What preserves the genuine gauge invariance is the correlation function of the genuine gauge invariant operator evaluated for such quantum 
states. ${ }^{11}$ While the relation between the dilatation invariance and the cancellation of the IRdiv has been discussed in a number of literatures, e.g., in refs. [39, 40, 44, 60, 63, 65-69], this aspect has not been clearly described elsewhere.

By contrast, since the curvature perturbation $\zeta_{\boldsymbol{k}}$ is not a genuine gauge invariant operator, the correlation functions which includes the operator $\zeta_{\boldsymbol{k}}$ suffers from the IRdiv (and also IRsec and SG). Because of that, the correlation functions $\left\langle\zeta_{\boldsymbol{k}_{L}} O_{\boldsymbol{k}_{S 1}} \cdots O_{\boldsymbol{k}_{S n}}\right\rangle$ diverge due to the accumulation of the soft modes. Here, $O_{\boldsymbol{k}_{S}}$ is either $\zeta_{\boldsymbol{k}_{S}}$ or $O_{\boldsymbol{k}_{S}}$. In order to make these correlation functions finite, we need to somehow introduce IR regularization. However, recall that a naive introduction of the IR cutoff violates the dilatation invariance, which was the starting point of the discussion about the consistency relation. Therefore, to be precise, we should not understand the consistency relation as the relation between the correlation functions for the Heisenberg operators $\zeta_{\boldsymbol{k}_{L}} O_{\boldsymbol{k}_{S 1}} \cdots O_{\boldsymbol{k}_{S n}}$ and $O_{\boldsymbol{k}_{S 1}} \cdots O_{\boldsymbol{k}_{S n}}$, since they are not well-defined. Instead, the consistency relation we discussed in this paper should be understood as the relation between the "correlation function" for the hard modes $O_{\boldsymbol{k}_{S 1}} \cdots O_{\boldsymbol{k}_{S n}}$ without any propagation of the soft modes and the one with the additional insertion of the free soft mode as an external leg. Then, both of them do not contain the loop corrections of the soft modes, which can lead to the IR enhancements. This is the reason why we needed to employ the approximation $\stackrel{\text { s.l. }}{\approx}$ in deriving the consistency relation.

\section{Relevance and irrelevance of soft graviton insertion}

The curvature perturbation $\zeta$ and the graviton $\gamma_{i j}$ are both massless fields and they have similar IR behaviours. In this section, we briefly show that the discussion about the consistency relation and the IR divergence for the graviton proceed almost in parallel to those for the curvature perturbation $\zeta$. For this purpose, we consider the shear transformation, which is a large gauge transformation:

$$
x^{i} \rightarrow \tilde{x}^{i} \equiv\left[e^{\frac{S}{2}}\right]_{j}^{i} x^{j},
$$

where $S_{i j}$ is a constant symmetric and traceless tensor. Under this large gauge transformation, the spatial metric transforms as

$$
\left[e^{\tilde{\gamma}(t, \tilde{\boldsymbol{x}})}\right]_{i j}=\left[e^{-\frac{S}{2}}\right]_{i} k\left[e^{-\frac{S}{2}}\right]_{j}^{l}\left[e^{\gamma(t, \boldsymbol{x})}\right]_{k l} .
$$

At the linear perturbation, $\gamma_{i j}$ is shifted as $\tilde{\gamma}_{i j}=\gamma_{i j}-S_{i j}$. The classical action is invariant under the large gauge transformation (5.1).

\footnotetext{
${ }^{11}$ Although the locality condition is necessary condition for the absence of IRdiv, this does not immediately imply that the locality condition is a requirement for the quantum state of the whole universe. When we discuss observables for a local observer, it would be allowed to trace out the degrees of freedom which the observer cannot see. After tracing out these degrees of freedom, the density matrix of the universe will be block diagonalized with a good precision. Then, the observables will correspond to the expectation values just for one of the blocks in the density matrix. In this sense, the actual observables are likely to be quite different from the simple expectation values for a given wave functional of the whole universe.
} 
Similar to the dilatation, we define the Noether charge for the large gauge transformation (5.1) as

$$
Q_{\gamma} \equiv \frac{1}{2} \int d^{3} \boldsymbol{x}\left[\Delta_{S} \gamma_{i j}(t, \boldsymbol{x}) \pi_{\gamma}^{i j}(t, \boldsymbol{x})+\pi_{\gamma}^{i j}(t, \boldsymbol{x}) \Delta_{S} \gamma_{i j}(t, \boldsymbol{x})\right],
$$

where $\pi_{\gamma}^{i j}$ denotes the conjugate momentum of $\gamma_{i j}$ and

$$
\Delta_{S} \gamma_{i j}(t, \boldsymbol{x}) \equiv \tilde{\gamma}_{i j}(t, \boldsymbol{x})-\gamma_{i j}(t, \boldsymbol{x}) .
$$

The invariance of the quantum state under this transformation requires

$$
Q_{\gamma}|\Psi\rangle=0 \text {. }
$$

Repeating a similar argument, we find that the invariance is preserved, when the following conditions

$$
\begin{aligned}
\frac{\partial}{\partial \bar{\gamma}^{c}}\left|\psi\left(\bar{\gamma}^{c}\right)\right| & =0, \\
i Q_{\gamma}|\Psi\rangle_{\bar{\gamma}^{c}} & =S_{i j} \frac{\partial}{\partial \bar{\gamma}_{i j}^{c}}|\Psi\rangle_{\bar{\gamma}^{c}},
\end{aligned}
$$

are satisfied. Here, $\bar{\gamma}_{i j}$ denotes the homogeneous mode of $\gamma_{i j}$ and $|\Psi\rangle_{\bar{\gamma}^{c}}$ denotes the projected quantum state into the eigenstate of $\bar{\gamma}_{i j}^{c}$.

Inserting the window function $W(\boldsymbol{x})$ into the integrand of the Noether charge $Q_{\gamma}$ and performing the Fourier transformation, we define $Q_{\gamma}^{W}\left(\boldsymbol{k}_{L}\right)$, which inserts the soft graviton $\gamma_{i j, \boldsymbol{k}_{L}}$. Again, we find that when the condition (5.7) can be extended to the soft modes with $\boldsymbol{k}_{L} \neq 0$, i.e.,

$$
i Q_{\gamma}^{W}\left(\boldsymbol{k}_{L}\right)|\Psi\rangle_{\tilde{\gamma}_{-\boldsymbol{k}_{L}}^{c}}=\left(2 \pi L_{c}\right)^{3} S_{i j-\boldsymbol{k}_{L}} \frac{\partial}{\partial \tilde{\gamma}_{i j-\boldsymbol{k}_{L}}^{c}}|\Psi\rangle_{\tilde{\gamma}_{-\boldsymbol{k}_{L}}^{c}}
$$

with $S_{i j \boldsymbol{k}_{L}} \equiv \hat{W}\left(\boldsymbol{k}_{L}\right) S_{i j}$, we obtain the consistency relation which relates the correlation functions for the hard modes and those with single insertion of the Gaussian soft graviton (see, e.g., refs. $[14,70]$ ). The quantum state $|\Psi\rangle$ changes due to the single insertion of the soft graviton, i.e.,

$$
Q_{\gamma}^{W}\left(\boldsymbol{k}_{L}\right)|\Psi\rangle \neq 0
$$

because the wave function is not completely flat in the direction of the soft graviton $\gamma_{i j} \boldsymbol{k}_{L}$ with $\boldsymbol{k}_{L} \neq 0$ in contrast to the homogeneous mode, whose wave function is completely flat, satisfying eq. (5.6). Let us emphasize again that this is not a spontaneous symmetry breaking, because the large gauge transformation (5.1) with an inhomogeneous $S_{i j}$ is not a symmetry of the classical action.

The correlation function of the genuine gauge invariant operator ${ }^{g} R$ is insensitive to the insertion of the soft graviton. This ensures the absence of the IRdiv due to the soft graviton in the correlation functions of ${ }^{g} R$ evaluated for $|\Psi\rangle$. By contrast, when we evaluate a correlation function for an operator which does not preserve the genuine gauge invariance, the insertion of the soft graviton changes the correlation function and this can lead to a break down of the perturbative expansion [71, 73]. (See also ref. [74].) 


\section{Concluding remarks}

The relation among the large gauge transformations, the consistency relations for the soft modes of $\zeta$ and $\gamma_{i j}$, their conservation in time, and the IR enhancements has been discussed in a number of literatures. However, as far as we understand, this relation was not fully clarified and it was sometimes understood in a misleading manner. The purpose of this paper is to sharpen the argument about the relation among these four. The invariance of the quantum state $|\Psi\rangle$ under the dilatation and shear transformations can be preserved, when the following two conditions are fulfilled. First, the amplitude of the wave function $\psi$ should be flat towards the directions for the homogeneous modes of $\zeta$ and $\gamma_{i j}$. Second, operating the Noether charges $Q_{\zeta}$ and $Q_{\gamma}$ on the quantum state of hard modes is equivalent to additively and time-independently shifting the homogeneous modes $\bar{\zeta}$ and $\bar{\gamma}_{i j}$, which interact with the hard modes, as described in eqs. (2.29) and (5.7).

The invariance under these large gauge transformations, just itself, leads to neither the consistency relation nor the absence of IRdiv. The additional conditions (2.29) and (5.7) are the non-trivial extensions to those which describe the insertion of the soft modes $\boldsymbol{k}_{L}(\neq 0)$, which are not always satisfied. These conditions can be interpreted as the locality condition, which states that the inhomogeneous dilatation and shear transformations only change the values of $\zeta$ and $\gamma_{i j}$ within each local universe. Since the wave function is not flat in the directions of the soft modes for the curvature perturbation and the graviton, the quantum state $|\Psi\rangle$ changes due to the insertion of these soft modes. When the locality conditions (2.39) and (5.8) are satisfied, the influence of these soft modes are described by the well-known consistency relations under the Gaussian approximation of the wave function for the soft modes. This argument also applies in deriving the consistency relation for massive fields. We also showed that the locality condition (2.39) implies the conservation of $\zeta$ in the soft limit within the perturbation theory. The same argument also applies to $\gamma_{i j}$.

The final issue is the IR enhancements due to the soft modes of $\zeta$ and $\gamma_{i j}$. In contrast to the correlation functions for $\zeta$ and $\gamma_{i j}$, which are not genuinely gauge invariant, the correlation functions for a genuine gauge invariant operator remain invariant under the insertion of the soft modes for the curvature perturbation $\zeta$ and the graviton $\gamma_{i j}$, when the locality conditions hold. This ensures the absence of the IRdiv due to the soft modes of $\zeta$ and $\gamma_{i j}$. In this paper, we did not discuss the IRsec and the SG, which yields the secular growth. When the locality conditions hold at each time slicing, repeating the same argument as in refs. [44, 64], we can show the absence of the IRsec and SG. In fact, this is the case when we choose the adiabatic vacuum (or the Euclidean vacuum) as the initial state of the universe.

Recently, the relation among the asymptotic symmetry, the soft theorem, and the IR divergence was discussed about gauge theories in asymptotically flat spacetime [26-28, 32]. In ref. [26], it was shown that the Weinberg's soft theorem for the soft photons and gravitons can be derived as the Ward-Takahashi identities for the asymptotic symmetry. (For a recent review, see ref. [29].) In ref. [32], the relation between the asymptotic symmetry and the IR divergence of the QED was discussed. About the IR divergence in QED, Faddeev and Kulish showed that the IR finiteness can be guaranteed, when we consider the dressed 
charged particles by soft photon clouds. (See also refs. [76, 77].) While there is a qualitative difference between the in-out formalism in QED and the in-in formalism in cosmology, our genuine gauge invariant operator ${ }^{g} R$, whose correlators can be IR finite with an appropriate choice of the initial state, also dresses the clouds of the soft $\zeta$ and $\gamma_{i j}$ as external legs.

At first glance, the IR structures for the gauge fields in the asymptotically flat spacetime have a certain similarity to those for the primordial perturbations $\zeta$ and $\gamma_{i j}$. However, a closer look may also reveal some differences in these two cases. For the gauge theories in the asymptotically flat spacetime, we are interested in the transition at the asymptotic infinity between before and after the propagations of the soft photons and gravitons. On the other hand, for the primordial perturbations, the asymptotic infinity is the spatial infinity and is out of reach in a causal evolution. Instead, what we are interested in is a locally defined quantity like an actually observable quantity. Moreover, as is summarized above, in order to derive the consistency relation, the soft theorem for $\zeta$ and $\gamma_{i j}$, we need to assume the locality condition, which is not trivially satisfied even in a Diff invariant theory, at least in the current gauge choice (the Maldacena gauge [14]). These give qualitative differences from the gauge theories in the asymptotically flat spacetime, which trivially satisfy the locality.

\section{Acknowledgments}

We would like to thank M. Mirbabayi for a fruitful conversation. T.T. was supported in part by JSPS KAKENHI Grant Number JP 17H06357, 17H06358, 24103001 and 24103006, and 15H02087. Y.U. is supported by JSPS KAKENHI Grant Number JP 26887018, 16K17689, and 16H01095. This research is supported in part by Building of Consortia for the Development of Human Resources in Science and Technology and the National Science Foundation under Grant No. NSF PHY11-25915 and also by DAIKO FOUNDATION.

\section{A Perturbative and non-perturbative definition of Noether charge}

In this section, we show that performing the dilatation and performing the perturbative expansion are not commutable processes, i.e., the dilatation transformation in the Heisenberg picture and the one in the interaction picture lead to different expressions. In order to see this, let us first consider the dilatation in the Heisenberg picture with the use of the Noether charge $Q_{\zeta}$ as

$$
\zeta_{s}(x)=e^{i Q_{\zeta}} \zeta(x) e^{-i Q_{\zeta}}=\zeta(x)+\Delta_{s} \zeta(x) .
$$

Then, we perturbatively expand both $\zeta(x)$ and $\zeta_{s}(x)$, i.e., before and after the dilatation transformation, following the standard procedure, as

$$
\zeta(t, \boldsymbol{x})=U^{I \dagger}(t) \zeta^{I}(t, \boldsymbol{x}) U^{I}(t)
$$

and

$$
\zeta_{s}(t, \boldsymbol{x})=U_{s}^{I \dagger}(t) \zeta_{s}^{I}(t, \boldsymbol{x}) U_{s}^{I}(t)
$$


where $U^{I}$ and $U_{s}^{I}$ denote the unitary operators which relate the Heisenberg and interaction picture fields for before and after the dilatation transformation. Using the interaction Hamiltonian $H^{I} \equiv H-H_{0}$, the unitary operator is given by

$$
U^{I}(t) \equiv T e^{-i \int^{t} d t^{\prime} H^{I}}, \quad U_{s}^{I}(t) \equiv T e^{-i \int^{t} d t^{\prime} H_{s}^{I}} .
$$

Since the free Hamiltonian $H_{0}$ changes due to the dilatation transformation while the total Hamiltonian does not, the interaction Hamiltonian $H^{I}$ and $U^{I}$ also change through the dilatation, i.e.,

$$
U_{s}^{I \dagger}(t) U^{I}(t)=1+\mathcal{O}(s) .
$$

Next, we show that the interaction picture field $\zeta_{s}^{I}(t, \boldsymbol{x})$, which is related to $\zeta_{s}$ as in eq. (A.3), does not coincide with

$$
\tilde{\zeta}_{s}^{I}(x) \equiv e^{i Q_{s}^{I}} \zeta_{I}(x) e^{-i Q_{s}^{I}}=\zeta_{I}(x)+\Delta \zeta_{I}(x),
$$

which is given by performing the dilatation transformation in the interaction picture. Here, $\Delta \zeta_{I}$ is given by replacing the Heisenberg fields with the interaction picture fields in $\Delta \zeta(x)$. Notice that $\tilde{\zeta}_{s}^{I}(x)$ is related to $\zeta_{s}(x)$ by the unitary operator $U^{I}(t)$, i.e.,

$$
\tilde{\zeta}_{s}^{I}(x)=U^{I}(t) \zeta_{s}(t, \boldsymbol{x}) U^{I \dagger}(t)
$$

while the standard perturbative prescription in the frame after the dilatation transformation uses the interaction picture field given by

$$
\zeta_{s}^{I}(t, \boldsymbol{x})=U_{s}^{I}(t) \zeta_{s}(t, \boldsymbol{x}) U_{s}^{I \dagger}(t) .
$$

As is shown in eq. (A.5), since the unitary operator $U_{I}(t)$ changes under the dilatation transformation, we obtain

$$
\tilde{\zeta}_{s}^{I}(x)-\zeta_{s}^{I}(x)=\mathcal{O}(s) .
$$

Therefore, the dilatation transformation in the interaction picture (A.6) does not give the interaction picture field defined in the standard prescription of perturbation theory after the dilatation transformation, i.e., eq. (A.3). This discrepancy vanishes by sending $s$ to 0 .

\section{B Computing the effective action}

In this appendix, we derive the expression of $W_{\delta g_{a_{1}} \cdots \delta g_{a_{n}}}^{(n)}\left(x_{1}, \cdots, x_{n}\right)$, defined in eq. (3.17). The linear term in the effective action is given by

$$
i S_{\mathrm{eff}(1)}^{\prime}=\sum_{a= \pm} \int d^{d+1} x \delta g_{a}(x) W_{\delta g_{\alpha}}^{(1)}(x),
$$

where $W_{\delta g_{\alpha}}^{(1)}$ is given by the expectation value as

$$
W_{\delta g_{+}}^{(1)}(x)=-W_{\delta g_{-}}^{(1)}(x)=\left\langle\left.\frac{\delta i S_{\chi}}{\delta g(x)}\right|_{\delta g=0}\right\rangle .
$$


Next, we compute the quadratic terms in $S_{\text {eff }}^{\prime}$. Taking the second variation of $S_{\text {eff }}^{\prime}$ with respect to $\delta g_{+}$, we obtain

$$
\begin{aligned}
W_{\delta g_{+} \delta \tilde{g}_{+}}^{(2)}\left(x_{1}, x_{2}\right)= & i^{2}\left\langle\left.\left.\frac{\delta S_{\chi}\left[\delta g_{+}, \boldsymbol{\chi}_{+}\right]}{\delta g_{+}\left(x_{1}\right)}\right|_{\delta g_{+}=0} \frac{\delta S_{\chi}\left[\delta g_{+}, \boldsymbol{\chi}_{+}\right]}{\delta \tilde{g}_{+}\left(x_{2}\right)}\right|_{\delta g_{+}=0}\right\rangle_{ \pm} \\
& +i \delta\left(x_{1}-x_{2}\right)\left\langle\left.\frac{\delta^{2} S_{\chi}\left[\zeta_{+}, \boldsymbol{\chi}_{+}\right]}{\delta g_{+}\left(x_{1}\right) \delta \tilde{g}_{+}\left(x_{1}\right)}\right|_{\delta g_{+}=0}\right\rangle_{ \pm},
\end{aligned}
$$

where $\delta g$ and $\delta \tilde{g}$ are either $\delta N, N_{i}, \zeta$, or $\gamma_{i j}$. Here, we introduced the expectation value:

$$
\left\langle O\left[\chi_{+}, \chi_{-}\right]\right\rangle_{ \pm} \equiv \frac{\int D \chi_{+} \int D \chi_{-} O\left[\chi_{+}, \chi_{-}\right] e^{i S_{\chi}\left[0, \chi_{+}\right]-i S_{\chi}\left[0, \chi_{-}\right]}}{\int D \chi_{+} \int D \chi_{-} e^{i S_{\chi}\left[0, \chi_{+}\right]-i S_{\chi}\left[0, \chi_{-}\right]}}
$$

Since the action $S_{\chi}\left[\delta g_{+}, \chi_{+}\right]$includes only local terms, the variation of $S_{\chi}\left[\delta g_{+}, \chi_{+}\right]$with respect to $\delta g_{+}\left(x_{1}\right)$ and $\delta \tilde{g}_{+}\left(x_{2}\right)$ yields the delta function $\delta\left(x_{1}-x_{2}\right)$ in eq. (B.3). Similarly, the second variation of $S_{\text {eff }}^{\prime}$ with respect to $\delta g_{-}$is given by

$$
\begin{aligned}
W_{\delta g_{-} \delta \tilde{g}_{-}}^{(2)}\left(x_{1}, x_{2}\right)= & i^{2}\left\langle\left.\left.\frac{\delta S_{\chi}\left[\delta g_{-}, \boldsymbol{\chi}_{-}\right]}{\delta g_{-}\left(x_{1}\right)}\right|_{\delta g_{-}=0} \frac{\delta S_{\chi}\left[\delta g_{-}, \boldsymbol{\chi}_{-}\right]}{\delta \tilde{g}_{-}\left(x_{2}\right)}\right|_{\delta g_{-}=0}\right\rangle_{ \pm} \\
& -i \delta\left(x_{1}-x_{2}\right)\left\langle\left.\frac{\delta^{2} S_{\chi}\left[\delta g_{-}, \boldsymbol{\chi}_{-}\right]}{\delta g_{-}\left(x_{1}\right) \delta \tilde{g}_{-}\left(x_{1}\right)}\right|_{\delta g_{-}=0}\right\rangle_{ \pm} .
\end{aligned}
$$

Taking the derivative with respect to both $\delta g_{+}$and $\delta g_{-}$, we obtain

$$
W_{\delta g_{+} \delta \tilde{g}_{-}}^{(2)}\left(x_{1}, x_{2}\right)=-i^{2}\left\langle\left.\left.\frac{\delta S_{\chi}\left[\delta g_{+}, \chi_{+}\right]}{\delta g_{+}\left(x_{1}\right)}\right|_{\delta g_{+}=0} \frac{\delta S_{\chi}\left[\delta g_{-}, \boldsymbol{\chi}_{-}\right]}{\delta \tilde{g}_{-}\left(x_{2}\right)}\right|_{\delta g_{-}=0}\right\rangle_{ \pm}
$$

and

$$
W_{\delta g_{-} \delta \tilde{g}_{+}}^{(2)}\left(x_{1}, x_{2}\right)=-i^{2}\left\langle\left.\left.\frac{\delta S_{\chi}\left[\delta g_{-}, \chi_{-}\right]}{\delta g_{-}\left(x_{1}\right)}\right|_{\delta g_{-}=0} \frac{\delta S_{\chi}\left[\delta g_{+}, \chi_{+}\right]}{\delta \tilde{g}_{+}\left(x_{2}\right)}\right|_{\delta g_{+}=0}\right\rangle_{ \pm}
$$

When the interactions of $\chi$ are perturbatively suppressed, we can compute the functions $W_{\delta g_{a_{1}} \delta \tilde{g}_{a_{2}}}^{(2)}\left(x_{1}, x_{2}\right)$ by expanding them in terms of the free propagators for $\chi$.

Open Access. This article is distributed under the terms of the Creative Commons Attribution License (CC-BY 4.0), which permits any use, distribution and reproduction in any medium, provided the original author(s) and source are credited.

\section{References}

[1] J.A. Harvey, Magnetic monopoles, duality and supersymmetry, hep-th/9603086 [INSPIRE].

[2] S.G. Avery and B.U.W. Schwab, Noether's second theorem and Ward identities for gauge symmetries, JHEP 02 (2016) 031 [arXiv:1510.07038] [INSPIRE].

[3] S. Weinberg, Adiabatic modes in cosmology, Phys. Rev. D 67 (2003) 123504 [astro-ph/0302326] [INSPIRE]. 
[4] D. Wands, K.A. Malik, D.H. Lyth and A.R. Liddle, A New approach to the evolution of cosmological perturbations on large scales, Phys. Rev. D 62 (2000) 043527 [astro-ph/0003278] [INSPIRE].

[5] A.A. Starobinsky, Multicomponent de Sitter (Inflationary) Stages and the Generation of Perturbations, JETP Lett. 42 (1985) 152 [INSPIRE].

[6] D.S. Salopek and J.R. Bond, Nonlinear evolution of long wavelength metric fluctuations in inflationary models, Phys. Rev. D 42 (1990) 3936 [inSPIRE].

[7] M. Sasaki and E.D. Stewart, A general analytic formula for the spectral index of the density perturbations produced during inflation, Prog. Theor. Phys. 95 (1996) 71 [astro-ph/9507001] [INSPIRE].

[8] M. Sasaki and T. Tanaka, Superhorizon scale dynamics of multiscalar inflation, Prog. Theor. Phys. 99 (1998) 763 [gr-qc/9801017] [InSPIRE].

[9] K.A. Malik and D. Wands, Evolution of second-order cosmological perturbations, Class. Quant. Grav. 21 (2004) L65 [astro-ph/0307055] [INSPIRE].

[10] D.H. Lyth, K.A. Malik and M. Sasaki, A general proof of the conservation of the curvature perturbation, JCAP 05 (2005) 004 [astro-ph/0411220] [INSPIRE].

[11] D. Langlois and F. Vernizzi, Conserved non-linear quantities in cosmology, Phys. Rev. D 72 (2005) 103501 [astro-ph/0509078] [INSPIRE].

[12] L. Senatore and M. Zaldarriaga, The constancy of $\zeta$ in single-clock Inflation at all loops, JHEP 09 (2013) 148 [arXiv:1210.6048] [INSPIRE].

[13] V. Assassi, D. Baumann and D. Green, Symmetries and Loops in Inflation, JHEP 02 (2013) 151 [arXiv:1210.7792] [INSPIRE].

[14] J.M. Maldacena, Non-Gaussian features of primordial fluctuations in single field inflationary models, JHEP 05 (2003) 013 [astro-ph/0210603] [INSPIRE].

[15] P. Creminelli and M. Zaldarriaga, Single field consistency relation for the 3-point function, JCAP 10 (2004) 006 [astro-ph/0407059] [INSPIRE].

[16] K. Hinterbichler, L. Hui and J. Khoury, An Infinite Set of Ward Identities for Adiabatic Modes in Cosmology, JCAP 01 (2014) 039 [arXiv:1304.5527] [INSPIRE].

[17] W.D. Goldberger, L. Hui and A. Nicolis, One-particle-irreducible consistency relations for cosmological perturbations, Phys. Rev. D 87 (2013) 103520 [arXiv:1303.1193] [INSPIRE].

[18] L. Berezhiani and J. Khoury, Slavnov-Taylor Identities for Primordial Perturbations, JCAP 02 (2014) 003 [arXiv: 1309.4461] [INSPIRE].

[19] T. Tanaka and Y. Urakawa, Conservation of $\zeta$ with radiative corrections from heavy field, JCAP 06 (2016) 020 [arXiv: 1510.05059] [INSPIRE].

[20] J. Garriga and Y. Urakawa, Consistency relations and conservation of $\zeta$ in holographic inflation, JCAP 10 (2016) 030 [arXiv:1606.04767] [INSPIRE].

[21] N. Arkani-Hamed and J. Maldacena, Cosmological Collider Physics, arXiv:1503.08043 [INSPIRE].

[22] X. Chen and Y. Wang, Large non-Gaussianities with Intermediate Shapes from Quasi-Single Field Inflation, Phys. Rev. D 81 (2010) 063511 [arXiv:0909.0496] [INSPIRE]. 
[23] T. Noumi, M. Yamaguchi and D. Yokoyama, Effective field theory approach to quasi-single field inflation and effects of heavy fields, JHEP 06 (2013) 051 [arXiv:1211.1624] [INSPIRE].

[24] M. Mirbabayi and M. Simonović, Effective Theory of Squeezed Correlation Functions, JCAP 03 (2016) 056 [arXiv: 1507.04755] [INSPIRE].

[25] H. Lee, D. Baumann and G.L. Pimentel, Non-Gaussianity as a Particle Detector, JHEP 12 (2016) 040 [arXiv:1607.03735] [INSPIRE].

[26] A. Strominger, On BMS Invariance of Gravitational Scattering, JHEP 07 (2014) 152 [arXiv: 1312.2229] [INSPIRE].

[27] T. He, V. Lysov, P. Mitra and A. Strominger, BMS supertranslations and Weinberg's soft graviton theorem, JHEP 05 (2015) 151 [arXiv:1401.7026] [INSPIRE].

[28] A. Strominger and A. Zhiboedov, Gravitational Memory, BMS Supertranslations and Soft Theorems, JHEP 01 (2016) 086 [arXiv:1411.5745] [INSPIRE].

[29] A. Strominger, Lectures on the Infrared Structure of Gravity and Gauge Theory, arXiv: 1703.05448 [INSPIRE].

[30] S. Weinberg, Infrared photons and gravitons, Phys. Rev. 140 (1965) B516 [inSPIRE].

[31] A. Campoleoni, D. Francia and C. Heissenberg, On higher-spin supertranslations and superrotations, JHEP 05 (2017) 120 [arXiv: 1703.01351] [INSPIRE].

[32] D. Kapec, M. Perry, A.-M. Raclariu and A. Strominger, Infrared Divergences in QED, Revisited, arXiv:1705.04311 [INSPIRE].

[33] C. Cheung, P. Creminelli, A.L. Fitzpatrick, J. Kaplan and L. Senatore, The Effective Field Theory of Inflation, JHEP 03 (2008) 014 [arXiv:0709.0293] [INSPIRE].

[34] M.H. Namjoo, H. Firouzjahi and M. Sasaki, Violation of non-Gaussianity consistency relation in a single field inflationary model, EPL 101 (2013) 39001 [arXiv:1210.3692] [INSPIRE].

[35] X. Chen, H. Firouzjahi, M.H. Namjoo and M. Sasaki, A Single Field Inflation Model with Large Local Non-Gaussianity, EPL 102 (2013) 59001 [arXiv:1301.5699] [INSPIRE].

[36] L. Berezhiani and J. Khoury, On the Initial State and Consistency Relations, JCAP 09 (2014) 018 [arXiv: 1406.2689] [INSPIRE].

[37] E. Dimastrogiovanni, M. Fasiello, D. Jeong and M. Kamionkowski, Inflationary tensor fossils in large-scale structure, JCAP 12 (2014) 050 [arXiv:1407.8204] [INSPIRE].

[38] E. Dimastrogiovanni, M. Fasiello and M. Kamionkowski, Imprints of Massive Primordial Fields on Large-Scale Structure, JCAP 02 (2016) 017 [arXiv:1504.05993] [INSPIRE].

[39] Y. Urakawa and T. Tanaka, IR divergence does not affect the gauge-invariant curvature perturbation, Phys. Rev. D 82 (2010) 121301 [arXiv: 1007.0468] [INSPIRE].

[40] Y. Urakawa and T. Tanaka, Natural selection of inflationary vacuum required by infra-red regularity and gauge-invariance, Prog. Theor. Phys. 125 (2011) 1067 [arXiv:1009.2947] [INSPIRE].

[41] T. Tanaka and Y. Urakawa, Dominance of gauge artifact in the consistency relation for the primordial bispectrum, JCAP 05 (2011) 014 [arXiv:1103.1251] [INSPIRE].

[42] N. Kundu, A. Shukla and S.P. Trivedi, Constraints from Conformal Symmetry on the Three Point Scalar Correlator in Inflation, JHEP 04 (2015) 061 [arXiv:1410.2606] [INSPIRE]. 
[43] N. Kundu, A. Shukla and S.P. Trivedi, Ward Identities for Scale and Special Conformal Transformations in Inflation, JHEP 01 (2016) 046 [arXiv: 1507.06017] [INSPIRE].

[44] T. Tanaka and Y. Urakawa, Strong restriction on inflationary vacua from the local gauge invariance II: Infrared regularity and absence of secular growth in the Euclidean vacuum, PTEP 2013 (2013) 063E02 [arXiv:1301.3088] [INSPIRE].

[45] S. Endlich, A. Nicolis and J. Wang, Solid Inflation, JCAP 10 (2013) 011 [arXiv:1210.0569] [INSPIRE].

[46] L. Bordin, P. Creminelli, M. Mirbabayi and J. Noreña, Solid Consistency, JCAP 03 (2017) 004 [arXiv: 1701.04382] [INSPIRE].

[47] S. Deser and A. Waldron, Partial masslessness of higher spins in (A)dS, Nucl. Phys. B 607 (2001) 577 [hep-th/0103198] [INSPIRE].

[48] R.P. Feynman and F.L. Vernon Jr., The Theory of a general quantum system interacting with a linear dissipative system, Annals Phys. 24 (1963) 118 [INSPIRE].

[49] R.P. Feynman and A.R. Hibbs, Quantum Mechanics and Path Integrals, McGraw-Hill, New York (1965).

[50] N.C. Tsamis and R.P. Woodard, The Physical basis for infrared divergences in inflationary quantum gravity, Class. Quant. Grav. 11 (1994) 2969 [INSPIRE].

[51] N.C. Tsamis and R.P. Woodard, The quantum gravitational back reaction on inflation, Annals Phys. 253 (1997) 1 [hep-ph/9602316] [INSPIRE].

[52] M.S. Sloth, On the one loop corrections to inflation and the CMB anisotropies, Nucl. Phys. B 748 (2006) 149 [astro-ph/0604488] [INSPIRE].

[53] M.S. Sloth, On the one loop corrections to inflation. II. The Consistency relation, Nucl. Phys. B 775 (2007) 78 [hep-th/0612138] [INSPIRE].

[54] D. Seery, One-loop corrections to a scalar field during inflation, JCAP 11 (2007) 025 [arXiv:0707.3377] [INSPIRE].

[55] D. Seery, One-loop corrections to the curvature perturbation from inflation, JCAP 02 (2008) 006 [arXiv: 0707.3378] [INSPIRE].

[56] Y. Urakawa and K.-i. Maeda, One-loop Corrections to Scalar and Tensor Perturbations during Inflation in Stochastic Gravity, Phys. Rev. D 78 (2008) 064004 [arXiv:0801.0126] [INSPIRE].

[57] H. Kitamoto and Y. Kitazawa, Soft Graviton effects on Gauge theories in de Sitter Space, Phys. Rev. D 87 (2013) 124004 [arXiv:1204.2876] [INSPIRE].

[58] H. Kitamoto and Y. Kitazawa, Soft gravitational effects in Kadanoff-Baym approach, JHEP 10 (2013) 145 [arXiv: 1305.2029] [INSPIRE].

[59] T. Tanaka and Y. Urakawa, Loops in inflationary correlation functions, Class. Quant. Grav. 30 (2013) 233001 [arXiv:1306 .4461] [INSPIRE].

[60] Y. Urakawa and T. Tanaka, Influence on Observation from IR Divergence during Inflation. I., Prog. Theor. Phys. 122 (2009) 779 [arXiv:0902.3209] [INSPIRE].

[61] Y. Urakawa and T. Tanaka, Influence on observation from IR divergence during inflation: Multi field inflation, Prog. Theor. Phys. 122 (2010) 1207 [arXiv:0904.4415] [InSPIRE]. 
[62] Y. Urakawa, Influence of gauge artifact on adiabatic and entropy perturbations during inflation, Prog. Theor. Phys. 126 (2011) 961 [arXiv:1105.1078] [INSPIRE].

[63] T. Tanaka and Y. Urakawa, Strong restriction on inflationary vacua from the local gauge invariance I: Local gauge invariance and infrared regularity, PTEP 2013 (2013) 083E01 [arXiv: 1209.1914] [INSPIRE].

[64] T. Tanaka and Y. Urakawa, Strong restriction on inflationary vacua from the local gauge invariance III: Infrared regularity of graviton loops, PTEP 2014 (2014) 073E01 [arXiv: 1402.2076] [INSPIRE].

[65] C.T. Byrnes, M. Gerstenlauer, A. Hebecker, S. Nurmi and G. Tasinato, Inflationary Infrared Divergences: Geometry of the Reheating Surface versus $\delta N$ Formalism, JCAP 08 (2010) 006 [arXiv: 1005.3307] [INSPIRE].

[66] M. Gerstenlauer, A. Hebecker and G. Tasinato, Inflationary Correlation Functions without Infrared Divergences, JCAP 06 (2011) 021 [arXiv:1102.0560] [INSPIRE].

[67] S.B. Giddings and M.S. Sloth, Semiclassical relations and IR effects in de Sitter and slow-roll space-times, JCAP 01 (2011) 023 [arXiv: 1005.1056] [INSPIRE].

[68] S.B. Giddings and M.S. Sloth, Cosmological observables, IR growth of fluctuations and scale-dependent anisotropies, Phys. Rev. D 84 (2011) 063528 [arXiv:1104.0002] [INSPIRE].

[69] L. Senatore and M. Zaldarriaga, On Loops in Inflation II: IR Effects in Single Clock Inflation, JHEP 01 (2013) 109 [arXiv: 1203.6354] [INSPIRE].

[70] L. Bordin, P. Creminelli, M. Mirbabayi and J. Noreña, Tensor Squeezed Limits and the Higuchi Bound, JCAP 09 (2016) 041 [arXiv: 1605. 08424] [INSPIRE].

[71] R.Z. Ferreira, M. Sandora and M.S. Sloth, Asymptotic Symmetries in de Sitter and Inflationary Spacetimes, JCAP 04 (2017) 033 [arXiv:1609.06318] [INSPIRE].

[72] A. Kehagias and A. Riotto, Inflation and Conformal Invariance: The Perspective from Radial Quantization, Fortsch. Phys. 65 (2017) 1700023 [arXiv:1701.05462] [INSPIRE].

[73] R.Z. Ferreira, M. Sandora and M.S. Sloth, Patient Observers and Non-perturbative Infrared Dynamics in Inflation, arXiv:1703.10162 [INSPIRE].

[74] J. Garriga and T. Tanaka, Can infrared gravitons screen Lambda?, Phys. Rev. D 77 (2008) 024021 [arXiv:0706. 0295] [INSPIRE].

[75] P.P. Kulish and L.D. Faddeev, Asymptotic conditions and infrared divergences in quantum electrodynamics, Theor. Math. Phys. 4 (1970) 745 [InSPIRE].

[76] V. Chung, Infrared Divergence in Quantum Electrodynamics, Phys. Rev. 140 (1965) B1110 [INSPIRE].

[77] D. Zwanziger, Reduction formulas for charged particles and coherent states in quantum electrodynamics, Phys. Rev. D 7 (1973) 1082 [InSPIRE]. 